\title{
Development of a validated LC- MS/MS method for the quantification of 19 endogenous asthma/COPD potential urinary biomarkers
}

\author{
Mona M. Khamis ${ }^{1}$, Darryl J. Adamko ${ }^{2}$ and Anas El-Aneed ${ }^{1}$ \\ ${ }^{1}$ College of Pharmacy and Nutrition, University of Saskatchewan, Saskatoon, SK, Canada. \\ ${ }^{2}$ Department of Pediatrics, College of Medicine, University of Saskatchewan, Saskatoon, SK, Canada.
}

\begin{abstract}
Obstructive airways inflammatory diseases sometimes show overlapping symptoms that hinder their early and correct diagnosis. Current clinical tests are tedious and are of inadequate specificity in special population such as the elderly and children. Therefore, we are developing tandem mass spectrometric (MS/MS) methods for targeted analysis of urine biomarkers. Recently, protonnuclear magnetic resonance $\left({ }^{1} \mathrm{H}-\mathrm{NMR}\right)$ analysis proposed 50 urinary metabolites as potential diagnostic biomarkers among asthma and chronic obstructive pulmonary disease (COPD) patients. Metabolites are divided into 3 groups based on chemical nature. For group 1 (amines and phenols, 19 urinary metabolites), we developed and validated a high performance liquid chromatographic (HPLC)-MS/MS method using differential isotope labeling with dansyl chloride. Method development included the optimization of the derivatization reaction, the MS/MS conditions, and the chromatographic separation. Linearity varied from 2 to $4800 \mathrm{ng} / \mathrm{mL}$ and the use of ${ }^{13} \mathrm{C}_{2}$-labeled derivatives allowed for the correction of matrix effects as well as the unambiguous confirmation of the identity of each metabolite in the presence of interfering isomers in urine. Despite the challenges associated with method validation, the method was fully validated as per the food and drug administration (FDA) and the European medicines agency (EMA) recommendations. Validation criteria included linearity, precision, accuracy, dilution integrity, selectivity, carryover, and stability. Challenges in selectivity experiments included the isotopic contributions of the analyte towards its internal standard (IS), that was addressed via the optimization of the IS concentration. In addition, incurred sample analysis was performed to ensure that results from patient samples are accurate and reliable. The method was robust and reproducible and is currently being applied in a cohort of asthma and COPD patient urine samples for biomarker discovery purposes.
\end{abstract}




\section{Introduction}

Asthma and chronic obstructive pulmonary disease (COPD) are a major cause of morbidity and they impose a huge economic burden with more than 600 million patients are currently diagnosed with these illnesses [1]. Despite the distinct differences in the pathophysiology of asthma and COPD, their diagnosis using currently available diagnostic tools is difficult in a typical primary care setting [2-6]. It is common for some patients to experience an overlap in the clinical presentations of asthma and COPD or even have both as co-existing conditions $[2,7,8]$. This is particularly valid with asthmatic smokers, elderly with normally declined lung functions, and those with more severe asthma [2-4]. Correct diagnosis is crucial as each disease has different therapeutic strategies [4]. Accordingly, with the inaccessibility of accurate diagnostic tools in typical primary care settings, the diagnosis of asthma and COPD is too often based on history as described by the patient. Therefore, recent work has focused on novel approaches to diagnosis.

Metabolomics is the study of the end products of cellular metabolism in healthy and diseased states

[9-12]. Applied metabolomics in medicine aims to link different biochemical pathways with disease diagnosis, severity, and therapeutic response [11, 12]. It has demonstrated promising outcomes in biomarker discovery and in personalized medicine $[11,13,14]$. Among the various biological fluids, urine is ideal for metabolomic analysis. Its collection is non-invasive and it has richer metabolite content in comparison to blood, saliva, exhaled breath condensate or bronchoalveolar lavage $[9,10]$. Beyond lung disease, urine metabolomics has been mostly studied for improving the detection of various types of cancer $[15,16]$, including that in the renal system $[17,18]$. Investigation of the urine metabolome has also included, but not limited to, the diagnosis of jaundice syndrome [19] and chronic heavy metal toxicity [20], the understanding of 
pathophysiology of depression [21] and the prediction of acute renal injury following cardiopulmonary bypass surgery in children [22].

Previous metabolomic studies on asthma or COPD subjects have been conducted in different biological fluids mostly in untargeted/semi-targeted approach $[9,10]$. For instance, a ${ }^{1} \mathrm{H}-\mathrm{NMR}$ study of COPD blood samples showed lower levels of alanine, valine and isoleucine in comparison to healthy individuals [23]. Using the same platform, exhaled breath condensate (EBC) of COPD patients revealed significantly lower levels of valine and lysine, while the levels of serine and tyrosine were significantly higher in comparison to controls [24]. An increase in arginine levels and a decrease in glutamine, valine and isoleucine levels were also observed in the serum of COPD patients $[25,26]$. On the other hand, the serum of asthmatic patients, using ${ }^{1} \mathrm{H}-\mathrm{NMR}$, showed low levels of arginine along with valine and alanine, whereas; high histidine and glutamine levels were observed [27]. It can be concluded from these studies, that amino acids metabolism is an important pathway in the pathogenesis of asthma and COPD.

Pertaining to urine, comparisons of the metabolomic profiles of healthy participants with either asthmatic [28, 29] or COPD subjects [30, 31] have been reported. Despite providing useful metabolomic information, these 'disease/no disease' studies are less interesting in clinical practice. People know when they have chronic trouble breathing. The more important question is knowing the cause or type of chronic lung disease and the type of treatment it requires. Accordingly, metabolomics investigating the disease severity in asthma or COPD shows more promise for translational and personalized medicine in primary care settings [32-34]. However, previous reports either involved few specific classes of metabolites (e.g. volatile metabolites) [32, 33] or resulted in the identity confirmation of very few metabolites [34]. 
To move to this important next stage, accurate targeted analysis is needed to clinically validate potentially therapeutic biomarkers. Contrary to the progress achieved in untargeted metabolomics, targeted quantification of endogenous metabolites is still at its infancy. Challenges in absolute quantification include the wide variations in metabolite concentration with diverse physicochemical properties [12]. In addition, neither endogenous metabolite- free matrices nor appropriate regulatory guidelines for the validation of bioanalytical methods for endogenous metabolites are available [35-37].

To the best of our knowledge, only 1 semi-targeted ${ }^{1} \mathrm{H}-\mathrm{NMR}$ study investigated the differences in the urine metabolome between asthma and COPD patients [38]. Based on this investigation, a group of urine metabolites were suggested as potential diagnostic biomarkers differentiating asthma and COPD. Before these ${ }^{1} \mathrm{H}-\mathrm{NMR}$ based metabolites can be used in a clinical lab, they require further validation. The suggested biomarkers were sub-divided into 3 groups based on their functional groups. Group 1 contains 19 metabolites that bear a primary amine, secondary amine or a phenolic group (Table 1). Group 2 contains 17 organic acids metabolites, whose identities and their validated quantification method will be described in a separate publication. Finally, group 3 contains 11 miscellaneous metabolites; including: quaternary ammonium compounds, sugars and nucleic acids as well as amino and organic acids that were not compatible with the developed methods for groups 1 and 2. Herein, we developed a fully validated LC-MS/MS method for the absolute quantification of a subset of 19 potential biomarker metabolites for the diagnosis of asthma and COPD. We adopted the deferential isotope labeling (DIL) approach [39] for the development of the quantification method. Amine and/or phenol functional groups contained within our metabolites can be derivatized with ${ }^{12} \mathrm{C}_{2} /{ }^{13} \mathrm{C}_{2}$-Dansyl chloride (DNS-Cl) as described by Guo et al. [39]. DIL has been mostly used for relative quantification purposes of the 
metabolome [39-42], however, despite the usefulness of published semi-quantitative studies using DIL-DNS-Cl, we are required by regulatory bodies a full validation of the analytical method since clinical data will be obtained. The presented work discusses the usefulness of DIL strategy for absolute biomarker quantification and approaches to address the challenges typically faced in endogenous quantitative metabolomics.

Table 1: Chemical structure of investigated metabolites of group 1

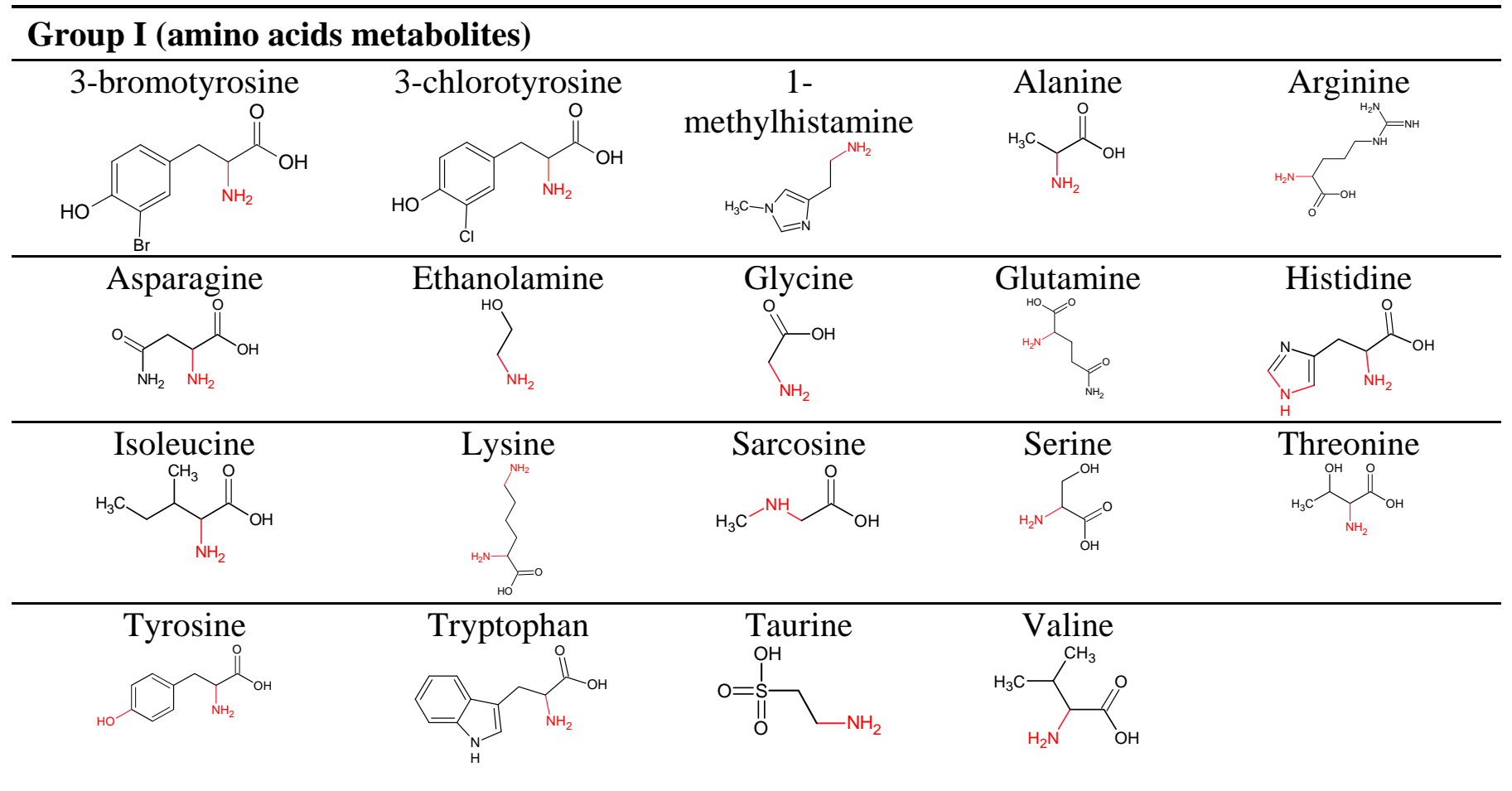

\section{Experimental}

\subsection{Materials and chemicals}

All chemicals were purchased from Sigma Aldrich (Oakville, ON, CA) unless otherwise stated. 3bromo tyrosine (98\%) was purchased from abcam (Cambridge, UK) and sarcosine (99.4\%) was 
purchased from Santa Cruz biotechnology (Santa Cruz, CA, USA). Optima® LC-MS grade acetonitrile (ACN) and water was purchased from Fischer Scientific (ON, CA). The concentration of creatinine within subject samples was determined using Jaffe's colorimetric reaction using QuantiChrom $^{\mathrm{TM}}$ creatinine assay kit (QC, CA) [43, 44].

\subsection{Synthesis of ${ }^{12} C_{2} /{ }^{13} C_{2}$-DNS-Cl}

${ }^{13} \mathrm{C}_{2}$-DNS-Cl bears $2-{ }^{13} \mathrm{C}$-methyl groups attached to the amino terminal within the naphthalene moiety of reagent. Structures of ${ }^{12} \mathrm{C}_{2} /{ }^{13} \mathrm{C}_{2}-\mathrm{DNS}-\mathrm{Cl}$ are compiled in Supplemental materials (Appendix I, Figure 1). The ${ }^{13} \mathrm{C}_{2}$-labeled reagent was not available commercially. Accordingly, it was synthesized in house along with the non-isotopic form in a 2-step reaction procedure $[39,45$, 46]. Synthesis protocol was modified from published methodologies in order to produce the highest yield of DNS-Cl (Supplemental materials (Appendix I, Figure 1)). The combination of the 2 step reaction is novel to the published methodology reported for the production of ${ }^{12} \mathrm{C}_{2} /{ }^{13} \mathrm{C}_{2}$ DNS-Cl in metabolomics [39]. The use of the commercially available ${ }^{12} \mathrm{C}_{2}-\mathrm{DNS}-\mathrm{Cl}$ was avoided because of its inadequate purity demonstrated by the precipitation of a white residual powder in $\mathrm{ACN}$ at the working concentration of the reagent. On the other hand, the 2 synthesized forms of DNS-Cl $\left({ }^{12} \mathrm{C}_{2}\right.$-and ${ }^{13} \mathrm{C}_{2}$-) were readily soluble in $\mathrm{ACN}$ without any insoluble matter, and were consequently used throughout the work.

\subsection{Patients characteristics}

Patients were enrolled after obtaining written informed consent, as approved by the University of Saskatchewan's biomedical research ethics board. Patients, seen at the Royal university hospital, Saskatoon, SK, were selected within an age group of 40-69 years old and were clinically assessed by a physician. Samples analyzed were age and gender matched as much as possible. No diet or 
time of collection restrictions were followed as the aim of the experiment was to identify metabolites that were sufficiently altered between asthma and COPD patient groups at a level that surpasses such variation. Urine samples collected from 42 patients were analyzed in this work. Patients' characteristics are as follows; gender (females: 59.5\%), body mass index (BMI: $\leq 25$; $45 \%, 25.1-30 ; 33 \%, 30.1-40 ; 14 \%$ and $>40 ; 12 \%)$, age ( $40-50$ years; $12 \%, 51-60$ years; $48 \%, 61-$ 69 years; $40 \%)$.

\subsection{Preparation of standard solutions}

A standard stock solution for each standard reference analyte was separately prepared at $3 \mathrm{mg} / \mathrm{mL}$ in 50\% ACN. For solubility/sensitivity aspects, the following exceptions were made; sarcosine and asparagine were prepared at $1.5 \mathrm{mg} / \mathrm{mL}$ in $50 \% \mathrm{ACN}$, tyrosine at $3 \mathrm{mg} / \mathrm{mL}$ in $0.1 \mathrm{M} \mathrm{HCl}$ and 3-bromotyrosine and 3-chlorotyrosine at $3 \mathrm{mg} / \mathrm{mL}$ in water.

\section{A. $\quad{ }^{12} \mathrm{C}_{2}$-derivatized analytes stock solution preparation}

Aliquots from the aforementioned analyte standard stock solutions were combined to prepare a standard working stock solution mixture of the 19 underivatized analytes at concentrations varying from 12.5 to $160 \mu \mathrm{g} / \mathrm{mL}$. The basic derivatization reaction was adopted from Dr. Liang Li's group, University of Alberta, Canada [39, 47]. However, changes to the experimental conditions were needed to optimize the reaction. The procedure was tailored to accommodate the introduction of the ${ }^{13} \mathrm{C}_{2}$-derivatized ISs mixture in calibration, validation (quality control, QC) solutions and patients' samples.

Briefly a volume of $50 \mu \mathrm{L}$ of the working stock solution was mixed with $30 \mu \mathrm{L}$ bicarbonate/carbonate buffer ( $\mathrm{pH} 9.4,0.5 \mathrm{M})$ and $40 \mu \mathrm{L}{ }^{12} \mathrm{C}_{2}$-DNS-Cl $(10.13 \mathrm{mg} / \mathrm{mL}$ in $\mathrm{ACN})$. The mixture was vortexed for $10 \mathrm{sec}$, spun down and placed in a thermostatically controlled water 
bath at $60^{\circ} \mathrm{C}$ for 30 minutes. Excess DNS-Cl was quenched through the addition of $10 \mu \mathrm{L} 0.25 \mathrm{M}$ $\mathrm{NaOH}$ with further heating at $60{ }^{\circ} \mathrm{C}$ for $10 \mathrm{~min}$. Seventy $\mu \mathrm{L}$ of $300 \mathrm{mM}$ formic acid (FA) in $50 \%$ ACN were added to acidify the medium and the resulting solution is referred to, further on, as the ${ }^{12} \mathrm{C}_{2}$-derivatized analytes stock solution. For the preparation of calibration standard solutions and QC samples, aliquots from the ${ }^{12} \mathrm{C}_{2}$-derivatized analytes stock solution were appropriately diluted in $50 \% \mathrm{ACN}$ to a volume of $40 \mu \mathrm{L}$ and spiked into $60 \mu \mathrm{L}$ of blank surrogate urine matrix containing $10 \mu \mathrm{L}{ }^{13} \mathrm{C}_{2}$-derivatized ISs solution. The mixtures were transferred into HPLC vials equipped with $100 \mu \mathrm{L}$ inserts for analysis.

B. $\quad{ }^{13} \mathrm{C}_{2}$-derivatized internal standards (ISs) preparation

${ }^{13} \mathrm{C}_{2}$-DNS-Cl $(10.13 \mathrm{mg} / \mathrm{mL}$ in $\mathrm{ACN})$ was used for the derivatization and the reaction mixture was completed to $250 \mu \mathrm{L}$ with $50 \%$ ACN. A volume of $10 \mu \mathrm{L}$ of the aforementioned solution introduced the optimized concentration of the ${ }^{13} \mathrm{C}_{2}$-derivatized ISs into the final mixtures.

\subsection{Urine preparation}

A. Blank surrogate urine matrix preparation

A pooled urine sample was prepared by mixing equal aliquots from 32 asthma patients, 6 COPD patients and 23 healthy controls. Pooled urine was stored in aliquots at $-80^{\circ} \mathrm{C}$ and derivatized as previously described using $\mathrm{ACN}$ instead of ${ }^{12} \mathrm{C}_{2}$-DNS-Cl. This matrix was used in the dilution of standard solutions, QC samples as well as urine samples for the quantification of highly abundant metabolites.

B. Patient's urine sample preparation 
Following collection, samples were promptly placed at $-80^{\circ} \mathrm{C}$. Within 1 week of expected sample analysis, samples were thawed to room temperature and divided into aliquots then refrozen at -80 ${ }^{\circ} \mathrm{C}$. At the time of the analysis; individual urine aliquots were allowed to thaw at room temperature and were 2 fold diluted with $\mathrm{ACN}$, vortexed and centrifuged at 13,000 rpm for $10 \mathrm{~min}$. A volume of $50 \mu \mathrm{L}$ of the supernatant solution was derivatized as previously described in 2.4.A. Following the addition of $300 \mathrm{mM} \mathrm{FA}$ in $50 \% \mathrm{ACN}, 50 \mu \mathrm{L}$ of the resulting solution were diluted into $50 \mu \mathrm{L}$ $50 \% \mathrm{ACN}$ containing $10 \mu \mathrm{L}{ }^{13} \mathrm{C}_{2}$-derivatized ISs. The final solution was transferred to an HPLC vial for analysis. For metabolites frequently present at high concentration (i.e. glycine, alanine, histidine and glutamine), derivatized urine samples were appropriately diluted with blank surrogate urine matrix. Fifty $\mu \mathrm{L}$ of the appropriate dilution were mixed with $50 \mu \mathrm{L}$ of $50 \%$ ACN containing $10 \mu \mathrm{L}{ }^{13} \mathrm{C}_{2}$-derivatized ISs.

\subsection{Instrumentation}

LC-MS/MS analysis was performed on a 1200 Agilent HPLC system (Mississauga, ON, Canada) interfaced to an AB Sciex 4000 API QTRAP instrument (AB Sciex, Concord, ON, Canada). The optimized instrumentation settings for chromatographic separation, derivatized analytes identification and quantification are compiled in Supplemental Tables (Table 1).

\subsection{Method Validation}

\section{A. Matrix effects and suitability of the blank surrogate urine}

We introduced the blank surrogate urine matrix approach to simulate derivatized urine and to be used in standards and QC samples preparation as well as in the dilution of highly concentrated patient samples. Matrix effects encountered from the blank surrogate urine matrix were evaluated according to equations 1,2 as recommended by the European medicines agency (EMA) [36] using 
high QC (HQC; 83\% of upper limit of quantification (ULOQ)) and low QC (LQC; $2.6 \times$ the lower limit of quantification, LLOQ) samples. Six replicates at low and high QC levels were prepared as described under ${ }^{12} \mathrm{C}_{2}$-derivatized analytes stock solution preparation and spiked into 6 different surrogate urine matrices prepared from 6 different sources of control urine according to the procedure in 2.5.A. The absolute peak areas of the derivatized analytes were compared to those of analogues QC samples prepared in 50\% ACN (i.e. neat solvent) $[35,36,48]$. The EMA also recommends the calculation of the CV\% of IS normalized matrix factor (MF), where a CV\% value of less than $15 \%$ indicates acceptable interferences from the matrix [36].

Equation 1: Matrix factor $(\mathrm{MF})=\mathrm{B} / \mathrm{A}$

Equation 2: IS normalized MF = MF analyte/MF IS

Where B is the absolute peak area of the analyte in surrogate urine matrix and A is the absolute peak area of the analyte in neat solvent $(50 \% \mathrm{ACN})$.

In addition, the U.S. Food and Drug Administration (FDA) recommends the comparison of the slopes of the calibration curves as a mean to evaluate matrix effects in ligand binding assays. [35] This approach is adopted to test the suitability of the surrogate blank urine as a valid substitute matrix for real derivatized urine. The methodological approach is described in details in Appendix II, supplementary materials.

\section{B. $\quad$ Selectivity}

Six double blanks containing only solvents and acidic/basic reagents (i.e., no analytes or DNS-Cl) were prepared and spiked into 6 blank surrogate matrices prepared from 6 different sources of 
control urine according to the procedure in 2.5.B. The interferences experienced at the retention times of the 19 derivatized metabolites or their analogues ${ }^{13} \mathrm{C}_{2}$-derivatized ISs were measured and the absolute peak areas were compared to that of the mean LLOQ according to equation 3. The method is deemed selective for an analyte and its ${ }^{13} \mathrm{C}_{2}$-derivatized IS if the interferences observed in the double blanks are less than $20 \%$ and $5 \%$ of LLOQ, respectively [36].

Equation 3: $=$ Standard or IS MRM channel $=\frac{\text { mean peak area of double blanks }}{\text { mean peak area of LLOQ }} \times 100$

\section{Carry over effects}

Carry over effects were investigated according to EMA guidelines [36]. The responses in blank solutions injected after highly concentrated samples were compared to that of the LLOQ. Carry over effects can be deemed insignificant if the interference observed in the subsequent blanks is less than $20 \%$ and $5 \%$ of LLOQ for the analyte and its corresponding IS, respectively [36].

\section{Linearity}

For the construction of 8 points-calibration curves, volumes from the ${ }^{12} \mathrm{C}_{2}$-derivatized analytes stock solution were serially diluted with 50\% ACN. Forty microliters of the appropriate dilution were mixed with $60 \mu \mathrm{L}$ blank surrogate urine matrix containing $10 \mu \mathrm{L}{ }^{13} \mathrm{C}_{2}$-derivatized ISs solution. Regression equations of the peak area ratios vs. the corresponding concentrations were generated using the least square regression model with a weighing factor of $1 / \mathrm{x}^{2}$. For accepting the calibration curve for each analyte; the back calculated concentration of the calibration points had to fall within $15 \%$ of the nominal concentration with the exception of the LLOQ which can tolerate 
up to $20 \%$ difference. At least $75 \%$ (6 out of 8 ) of the calibration points must fulfil this criterion $[35,36,49]$. The lower limit of quantification was determined according to the signal-to-noise ratio. The analyte response at the lowest end of the calibration curve should be at least 5 times higher than the signal encountered at the blank $[35,36]$.

\section{E. $\quad$ Intra- and inter- day accuracy and precision}

The intra- and inter-day accuracy and precision of the developed method were assessed, on 3 non consecutive days, using 4 QC levels covering the range of the calibration curves $[35,36,49]$. The HQC was set at $83 \%$ of the ULOQ. The middle QC (MQC) was set at $42 \%$ of the calibration range and the lower QC (LQC) was 2.6 times the concentration of the LLOQ. The LLOQ is the fourth level of the QC samples. QC samples were prepared from different stock solutions in 6 replicates, spiked into surrogate urine matrix and analyzed on 3 different days for the evaluation of inter-day accuracy and precision. The mean of the observed concentration must be within $15 \%$ of the nominal concentration for all levels with the exception of the LLOQ (within 20\%). In addition, it must not deviate from the nominal concentration more than $15 \%$ with the exception of the LLOQ in which a coefficient of variation (CV\%) of $20 \%$ is still acceptable $[35,36,49]$.

\section{F. $\quad$ Stability studies}

Stability was assessed using freshly prepared QC samples at multiple levels including benchtop stability, autosampler stability, freeze thaw stability, and short- and long-term stability. Details of the stability experiments are shown in Appendix III, supplementary materials.

\section{G. Dilution integrity}


An aliquot from ${ }^{12} \mathrm{C}_{2}$-derivatized analytes stock solution was spiked into surrogate urine matrix to produce a simulated urine sample in which the concentrations of the analytes are 6.7 folds higher than the ULOQ. Aliquots from this solution were 5, 10 and 20 fold diluted with blank surrogate urine and $50 \mu \mathrm{L}$ of each were mixed with $50 \mu \mathrm{L}$ of $50 \% \mathrm{ACN}$ containing $10 \mu \mathrm{L}$ of ${ }^{13} \mathrm{C}_{2}$-derivatized ISs. Patients' samples that were initially analyzed early in the method development phase and were found to contain analytes at concentrations above their ULOQ, especially for histidine, were used to evaluate the investigated dilution range. After derivatization, these patients' urine samples were diluted using blank surrogate urine matrix and reinjected into the system. This experiment was of particular importance in order to confirm the appropriateness of the tested dilution range before the application of the validated method on the study samples.

\section{H. $\quad$ Incurred sample analysis}

In addition to QC samples, the accuracy and precision of the developed method were also evaluated on patient urine samples. Four patient samples were selected so that they would contain very low, very high and middle creatinine values amongst the analyzed patient samples. Sample analysis was done over 2 consecutive days and a third day which was 2 weeks apart. The concentrations of the analytes obtained from their reanalysis should be within $20 \%$ of their mean for at least $67 \%$ of the repeats [36]. Since, guidelines adopt similar cut-off values for the acceptance of method accuracy and precision, a CV\% value of up to $20 \%$ was also deemed appropriate for the evaluation of the precision of the analysis.

\subsection{Analysis of patient urine samples}

Asthma and COPD patient urine samples were processed as described under 2.5.B. along with calibration and validation standards. The analytical run was started by equilibrating the instrument 
with triple injections of a HQC sample [50]. This was done to ensure the acceptable performance of the system before the analysis of a sample batch [50]. The batch was also ended by triple injection of the high QC sample to ensure system suitability throughout the batch [50]. Each batch consisted of double blank, blank, 8- point calibration set, 3 levels of QC samples prepared in duplicate and urine samples [35, 36]. To minimize any chances of carry over effects, samples were arranged ascendingly according to their creatinine values. Data from the QC samples provide the basis of accepting or rejecting a single run. At least $67 \%$ of the QC samples must fall within $15 \%$ of their respective nominal values. At least $50 \%$ of the QC samples at each level must fulfil this criterion $[35,36,49]$.

\section{Results and Discussion}

\subsection{Rationale for adopting DIL strategy}

DNS-Cl is a well-established derivatizing reagent that readily reacts with primary and secondary amines as well as phenols in alkaline medium [51]. It is, therefore, an ideal choice for the targeted 19 metabolites. Chemical derivatization enhances the chromatographic separation of such polar and low molecular weight metabolites on conventional reversed stationary phases (e.g. C18) [39]. In addition, derivatization unifies, to some extent, the physicochemical properties of the produced derivatives bearing the same signature dansyl moiety [39]. Derivatization can also improves ESI ionization [39-41] and the introduced tag, such as DNS shift low molecular weight metabolites by 234 Daltons out of the low $\mathrm{m} / \mathrm{z}$ region that typically exhibits high background noise, allowing for improved detectability [39].

Most importantly, DIL can address one of the major hurdles in absolute metabolite quantification that is the availability of suitable internal standards (IS). Structural analogues cannot guarantee the 
accuracy and precision of quantification $[39,52]$ and the use of isotopically-labeled ISs is challenging due to their price or commercial availability [39, 53]. DIL technique uses 2 isotopologues of a derivatizing reagent to produce light and heavy (deuterated or ${ }^{13} \mathrm{C}$-containing)derivatized metabolites. DIL using DNS-Cl allowed for the generation of a stable isotope IS for each target metabolite of the 19 targeted molecules, as discussed below.

\subsection{Method development}

\subsubsection{Optimization of the derivatization reaction and sample preparation}

Since the metabolites of interest are endogenous in nature; it was essential to optimize the reaction conditions in patient urine samples and in the standard mixture (data not shown). Each derivatization reaction condition was varied while keeping all other conditions constant. Heating temperature was tried at $45^{\circ} \mathrm{C}$ and $60^{\circ} \mathrm{C}$, while the heating time was investigated at $15,30,45$ and $60 \mathrm{~min}$. Both conditions were optimized at $60{ }^{\circ} \mathrm{C}$ and $30 \mathrm{~min}$, respectively. Finally the reagent volume was tried at 20,40,60 and $80 \mu \mathrm{l}$ and the volume was optimized at $40 \mu \mathrm{l}$. Heating the reaction mixture for a period of $60 \mathrm{~min}$ at $60^{\circ} \mathrm{C}$ as reported $[39,47]$ did not add any significant enhancement in the obtained responses, particularly, in the urine sample. In addition, no significant advantage was obtained by using the same volume from a relatively high concentrated ${ }^{12} \mathrm{C}_{2}$-DNSCl solution $(20 \mathrm{mg} / \mathrm{mL})$.

A volume of $30 \mu \mathrm{L}$ of $0.5 \mathrm{M}$ carbonate/bicarbonate buffer ( $\mathrm{pH} 9.4$ ), was sufficient to create the basic medium necessary for a reproducible dansylation reaction in urine and standard solutions. Higher volumes of the buffer were associated with an incompatible high salt content manifested as phase separation in the reaction mixtures. Although the basic method was based on published 
work $[39,47]$, the optimization experiments favored a shorter reaction time with much less reagent content, and consequently, less contamination/ion suppression in the mass spectrometer.

Another vital element of the derivatization reaction is the composition of the organic/aqueous medium. A medium of 54\% ACN was essential to prevent phase separation of the organic DNS$\mathrm{Cl}$ solution in the reaction mixture following derivatization. Interestingly, a similar observation of solvent incompatibility was reported along with the formation of an unknown precipitate in the reaction medium that contained 33\% ACN [42]. However, increasing the ACN content to 50\% was sufficient to overcome both issues [42].

For the optimization of patient samples preparation, urine was 1:1 diluted with ACN and centrifuged at 13,000 rpm for $10 \mathrm{~min}$. Enhancement in the analytical signals was not observed upon the filtration of the supernatant with 0.2 or $0.45 \mu \mathrm{m}$ PVDF filters (data not shown). Therefore, for the sake of simplicity and metabolite loss prevention, $50 \mu \mathrm{L}$ aliquots from the centrifuged supernatant were directly transferred into the reaction vial for derivatization.

Pertaining to the stoichiometry of the reaction; histidine and lysine bear $\underline{2}$ primary amino groups, while tyrosine and bromo- and chloro- derivatives of tyrosine bear a primary amine and phenolic functional groups. The reaction of these metabolites with DNS-Cl could proceed either in a 1:1 [54] or 1:2 [39] stoichiometric fashion. We found that, under the derivatization conditions described above, the mono tagged tyrosine compounds represented less than $10 \%$ of the double derivatized products. In the case of lysine, $70 \%$ of the derivatized entity was a double tagged species. Histidine formed an almost equal ratio of both forms. The consistency of the stoichiometric behavior was assured through 2 main observations; a) the derivatization optimization experiments and b) DNS-Cl hydrolytic product. 
The optimization of ${ }^{12} \mathrm{C}_{2}$-DNS-Cl concentration/volume confirmed that no improvements in the intensity of the double tagged species were observed with higher concentrations/volumes of the reagent. On the other hand, heating the reaction mixture for an additional $10 \mathrm{~min}$ period in the presence of $\mathrm{NaOH}$ resulted in the hydrolysis of excess DNS-Cl into dansyl hydroxide [51]. The presence of dansyl hydroxide, monitored at $\mathrm{m} / \mathrm{z}, 252.1>170.1$ in the injected standards and urine solutions confirmed that a remaining unreacted portion of DNS-Cl was indeed present at the end of the derivatization reaction and was successfully hydrolyzed with $\mathrm{NaOH}$. Based on the above findings, transitions corresponding to the double tagged species were selected for all of the aforementioned analytes bearing 2 potential reaction sites allowing for a $4.01 \mathrm{Da}$ difference between each analyte and its corresponding IS. In addition, the double tagged metabolites were of higher hydrophobicity and were consequently well separated from other mono-tagged metabolites. The enhanced ionization of the double tagged metabolites due to the higher organic solvent content needed for their elution allowed for higher sensitivity during quantification.

\subsubsection{Optimization of the mass spectrometric detection and quantification}

The MS/MS fragmentation pattern for each derivatized metabolite was generated and rationalized. Product ions observed from the collision induced dissociation (CID)-MS/MS of the derivatives contained the dansyl moiety and that was in agreement with the reported fragmentation behavior of the reagent [47].

The most abundant product ions for all metabolites are observed at $\mathrm{m} / \mathrm{z} 157.09$ and $\mathrm{m} / \mathrm{z}, 170.1$, corresponding to the methyl amino naphthalene and dimethylamino naphthalene charged moieties, respectively (Figure 1) [47]. In order to allow for adequate mass spectrometric separation at unit resolution between each analyte and its IS, it was favorable to maintain the isotopically labeled 
methyl groups within the selected MRM transitions to achieve a mass difference of $\underline{2}$ mass units. For this reason, the product ion at $m / z 170.1$ was selected as the quantifier transition for all analytes. The collision energy and declustering potential were optimized for each analyte for maximum generation of the quantifier product ion. Additionally, diagnostic product ions were identified, whenever possible, for each analyte to confirm its identity in patient urine samples (Supplemental Tables (Table 1)).

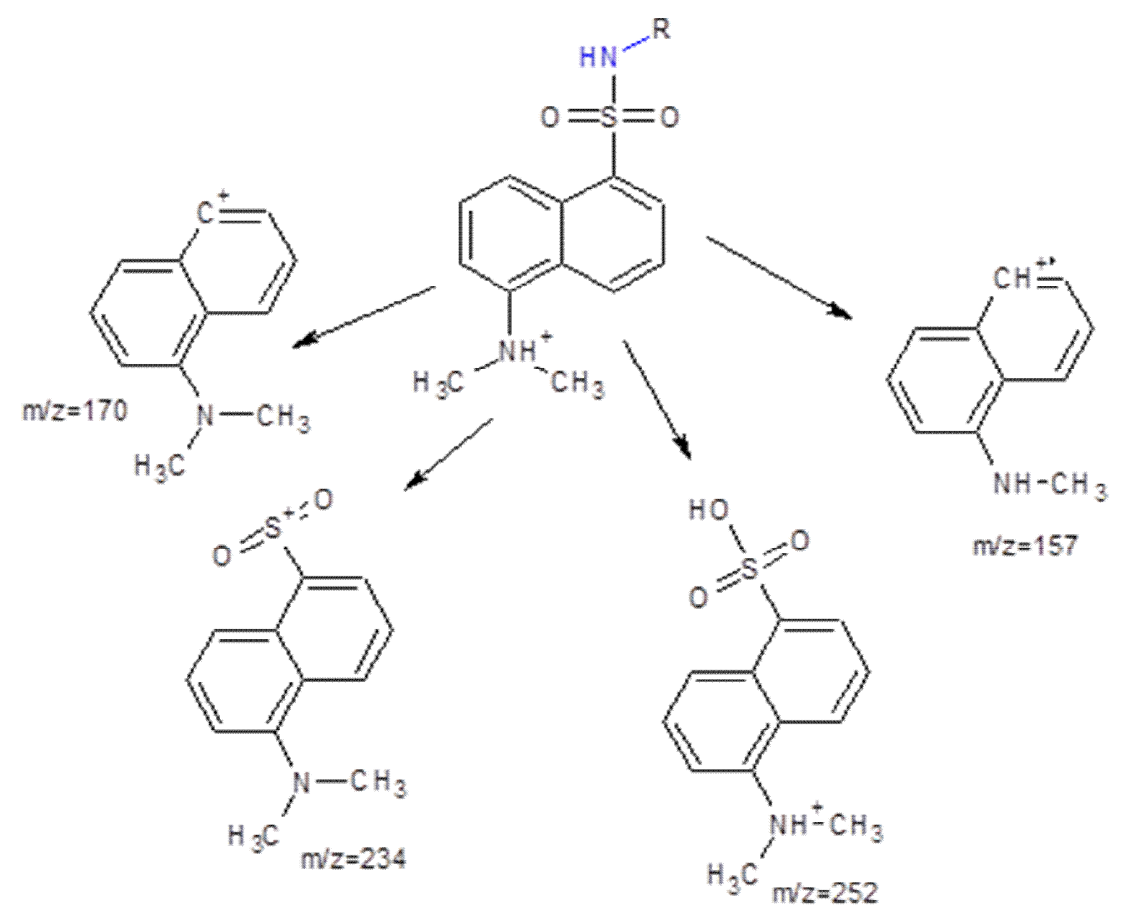

Figure 1: Schematic representation of the general fragmentation pattern of the dansylated analyte. 


\subsubsection{Optimization of the chromatographic separation}

Adequate separation of all analytes was achieved over 33 minute run using the optimized binary gradient mobile phase system (Figure 2). The presence of a perfectly co-eluting IS bearing ${ }^{13} \mathrm{C}_{2}$ carbons for each metabolite aided in its unambiguous identification in complex patient urine samples. For instance, urine samples contained multiple isomers of isoleucine that could also react with dansyl chloride. The secondary diagnostic product ion at $m / z 350$ was also present within the closest eluting peak to isoleucine which can be probably attributed to leucine (Supplemental materials, Appendix IV, Figure 2)). However, the combination of retention time matching with standard isoleucine peak in addition to pairing with ${ }^{13} \mathrm{C}_{2}$-derivatized IS added absolute confidence in its quantification. LC base-line resolved unidentified isomeric dansylated metabolites peaks were also noticed for alanine and arginine.

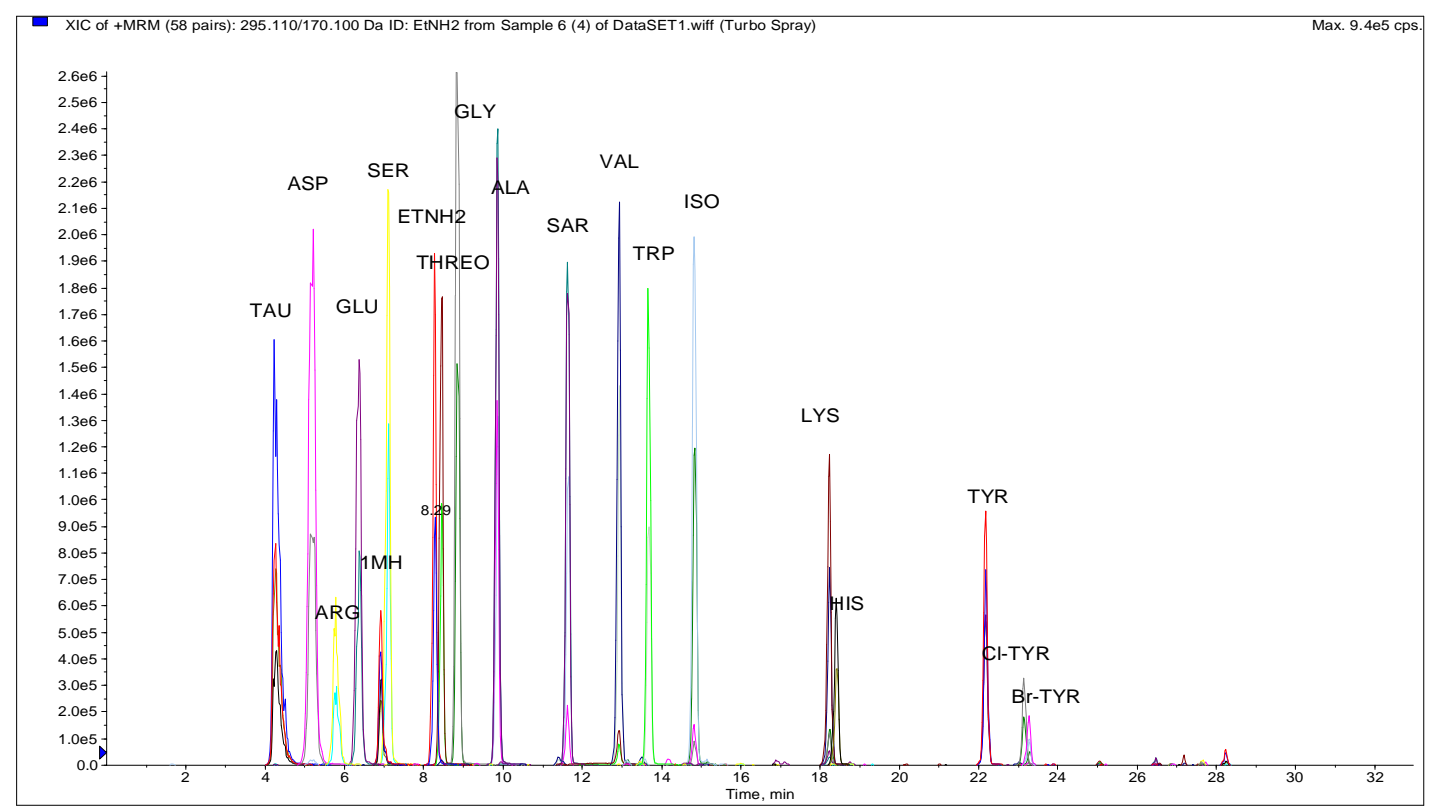

Figure 2: Representative extracted ion chromatogram of the optimized method for the quantification of 19 dansylated analytes in standard mixture. 


\subsection{Method validation}

The guidelines for bioanalytical method validation issued by the FDA [35, 49] and EMA [36] were followed. Despite being inclusive platforms for pharmaceutical/biomedical analyses, these guidelines were not always sufficient to address the challenges associated with endogenous metabolite quantification. In the following discussions; validation criteria of these guidelines are discussed along with atypical analytical/statistical approaches that were adopted to meet the unforeseen challenges identified during method development and validation.

\section{Evaluation of blank surrogate urine matrix suitability}

The FDA recommends the use of the same sample matrix for the construction of calibration curves and QC samples [35,49]. However, this is not feasible when conducting a metabolomic study, where the metabolites of interest are endogenously present in the blank matrix. With the absence of guidelines on the acceptable matrix for standard preparations in metabolomics, various strategies have been employed including the use of solvents of reconstitution, simulated and stripped matrices, biological fluids with low endogenous concentration of metabolites, standard addition method or deuterated surrogate analytes [37, 55-57].

On the other hand, underivatized urine was recently used as a surrogate matrix to which derivatized analytes were added [58]. The latter approach seemed, in principle, as the best strategy to adopt in this work. However, the derivatization procedure involved the addition of $\mathrm{Na}_{2} \mathrm{CO}_{3} / \mathrm{NaHCO}_{3}$ buffer, $\mathrm{NaOH}$ and FA that could change the nature of the urine (i.e. the matrix). Therefore, we introduced the surrogate blank urine matrix approach. Modified student t- test was used for the comparison between surrogate and derivatized matrices $[58,59]$. t-test values were always lower than $\mathrm{t}$-critical values at $p=0.05$ suggesting the insignificant difference between both matrices. 
Therefore, the use of the surrogate urine matrix provided the most accurate concentration within the real derivatized patient samples (Supplemental Tables (Table 2)).

\section{Evaluation of matrix effects}

The FDA states that the identification and elimination of matrix effects should be investigated during the development phase of an LC-MS/MS method, however, it does not provide any guidelines on the evaluation of such effects [35]. On the other hand, specific guidelines on the evaluation of matrix effects have been compiled by the EMA [36]. We found that the matrix factor was $96-130 \%$, where, the majority of analytes suffered from low ion enhancement effect (Supplemental Tables (Table 3)). This effect, not commonly seen in MS, was further confirmed through the comparison of the slopes of calibration curves generated in surrogate blank urine and neat solvent (50\% ACN) (Supplemental materials, Appendix V (Figure 3)). The vast variation in the obtained slopes further confirmed the unsuitability of the solvent as a substitute to the surrogate blank urine or derivatized urine samples (Supplemental materials, Appendix V, (Figure 3)).

The CV\% of IS normalized MF was lower than $15 \%$ in the developed method. In fact, the average values of the IS normalized MF were almost close to unity for all metabolites which indicates a very close similarity in the MS response between the analyte and it's IS, typically satisfied with the use of isotope coded IS (Supplemental Tables (Table 3)) [57]. As such, the elution of a pair of chemically and physically similar compounds provides the best correction of matrix effects encountered from the urine.

\section{$\underline{\text { Selectivity }}$}

Selectivity is described as the ability of the analytical method to unequivocally identify and quantify the analyte of interest in the presence of other components within a biological matrix [35, 36]. The FDA recommends the use of $\underline{6}$ different sources of the blank biological matrix to confirm 
the selectivity at the LLOQ. However, the acceptance values for a selective method were not suggested [35]. On the other hand, the EMA guidelines specify that within the double blanks, interferences should be less than $20 \%$ and $5 \%$ of LLOQ of the analyte and its corresponding IS, respectively [36].

As seen in Table 2, interferences encountered at the analyte and the IS channels for the 19 metabolites were found to be less than 14 and $2 \%$, respectively. If only these guidelines were to be followed, such results would be sufficient to confirm the selectivity of the developed method for the 19 metabolites $[35,36]$. However, due to the use of ${ }^{13} \mathrm{C}_{2}$-labeled internal standards that differ from their ${ }^{12} \mathrm{C}_{2}$ - counterparts by merely 2 mass units, other challenges were associated with the IS purity and its natural isotopic contribution. This necessitated extra analytical assessments which were not specifically mentioned in the currently available bioanalytical method validation protocols. Such challenge is expected as clinical metabolomics is relatively a new area of investigation with little reports available on validated methods for absolute quantification.

The first interference, IS purity, is expected to arise from any ${ }^{12} \mathrm{C}_{2}$ - DNS-Cl impurity in the ${ }^{13} \mathrm{C}_{2}-$ DNS-Cl that will eventually result in the false positive estimation of the analyte's concentration [60]. The ${ }^{12} \mathrm{C}_{2}$ - impurity within ${ }^{13} \mathrm{C}_{2}$-reagent would react in the IS reaction mixture giving rise to ${ }^{12} \mathrm{C}_{2}$-derivatized analyte. In addition, the amount of the produced ${ }^{13} \mathrm{C}_{2}$-derivatized IS would be less than the expected theoretical values. Being expressed as a ratio, the responses at the lower end of the calibration curve will be the most affected by any impurity of the used IS. In general, it can be argued that despite the absence of guidelines to evaluate the isotopic purity of DIL IS, the FDA and EMA recommendations can be still applicable if the ${ }^{12} \mathrm{C}_{2}$-impurities within the ${ }^{13} \mathrm{C}_{2}$ derivatized are treated as other interferences at the analyte's MRM channel. 
Accordingly, in order to address purity issues, the typical selectivity experiment was repeated, however, the double blanks were spiked with the optimized ${ }^{13} \mathrm{C}_{2}$-derivatized concentration with no ${ }^{12} \mathrm{C}_{2}$-analytes added. Contributions from ${ }^{12} \mathrm{C}_{2}$-impurities were then evaluated at the analytes channel. As shown in Table 2, these interferences were below $20 \%$ for all analytes; in fact all of them were below $12 \%$ with the vast majority below $7 \%$ (Table 2). This assessment confirms the adequate purity of the ${ }^{13} \mathrm{C}_{2}$-reagent and accordingly, the adequate purity of the IS-produced derivatives.

The second source of interference is the contribution of the $[\mathrm{M}+\mathrm{H}+2]^{+}$natural isotopic peak of the ${ }^{12} \mathrm{C}_{2}$-analyte on its IS's $\mathrm{Q}_{1} / \mathrm{Q}_{3}$ transition. This contribution is more critical for all metabolites bearing 1 dansyl tag as each IS differs from its ${ }^{12} \mathrm{C}_{2}$-analyte by 2 mass units. On the other hand, doubly derivatized ISs, namely, ${ }^{13} \mathrm{C}_{4}$-lysine, ${ }^{13} \mathrm{C}_{4}$-histidine, ${ }^{13} \mathrm{C}_{4}$-tyrosine, ${ }^{13} \mathrm{C}_{4}$-chloro- and ${ }^{13} \mathrm{C}_{4}$ bromo-tyrosine have adequate mass separation beyond the natural isotopic pattern of their analogues ${ }^{12} \mathrm{C}_{4}$-analytes (4.01 units).

For singly tagged compounds, the isotopic interference is more significant at the higher end of the calibration curve. At high concentrations of the analyte, its $[\mathrm{M}+\mathrm{H}+2]^{+}$isotopic peak concomitantly increases resulting in a false increase in the IS peak area also being integrated at the $[\mathrm{M}+\mathrm{H}+2]^{+}$ channel. Consequently, a possible false negative estimation of the analyte response, expressed as a ratio relative to the IS, is possible leading to a non-linear relationship (Supplemental materials, Appendix VI (Figure 4)). The isotopic contribution can be corrected either through subtraction or applying algorithm programs for deisotoping or via narrowing the linear range to avoid the significant contribution of the analyte at higher concentration [60-62]. Neither of these approaches were suitbale solution during the development/validation phase of this method, taking into account that a wide linear range was needed to cover the vast variation in concentrations among different 
metabolites. In addition, each metabolite can also considerably vary in concentration among urine samples depending on the hydration state of a patient. And for the sake of the method's simplicity, an extra correction step that would need an in-house computer program was sought to be avoided. Alternately, another approach was adopted in which the concentration of the IS is increased so that the effect of the isotopic contribution from its analyte become negligible [60]. Logically, the maximum possible isotopic contribution would be encountered at the ULOQ. For this reason, triplicates of the ULOQ were prepared without the inclusion of the IS and were analyzed. The mean response due to the contribution of the analyte's isotopic peak was calculated at the IS's channel. ISs spiked at concentrations equivalent to $66 \%$ of the ULOQ resulted in isotopic interferences less than 3\% in the IS's peak area which did not compromise the validity of method's linearly, accuracy and precision (Table 1, Supplemental materials, Appendix VI, (Figure 4)). 
Table 2: Selectivity of the developed method, purity and isotopic contribution assessment of the ${ }^{13} \mathrm{C}_{2}$ DNS-Cl

\begin{tabular}{|c|c|c|c|c|}
\hline & \multicolumn{2}{|c|}{ Selectivity } & \multirow{2}{*}{$\begin{array}{c}\text { Purity of } \\
{ }^{13} \mathrm{C}_{2}-\mathrm{DNS}-\mathrm{Cl} \\
\text { Mean \% Response } \\
\text { of blank at the } \\
\text { analyte channel } \pm \\
\mathrm{SD}^{2}\end{array}$} & \multirow{2}{*}{\begin{tabular}{|c|} 
Isotopic \\
contribution \\
Mean \% isotopic \\
contribution of the \\
ULOQ on the IS \\
channel $\pm \mathrm{SD}^{3}$ \\
\end{tabular}} \\
\hline Analyte & $\begin{array}{c}\text { Mean \% Response of } \\
\text { double blank at the } \\
\text { analyte channel } \pm \\
\text { SD }^{1}\end{array}$ & $\begin{array}{c}\text { Mean \% Response of } \\
\text { double blank at the IS } \\
\text { channel } \pm \mathrm{SD}^{1}\end{array}$ & & \\
\hline SAR & $5.34 \pm 2.51$ & $0.79 \pm 0.47$ & $6.3 \pm 0.7$ & $1.10 \pm 0.31$ \\
\hline $1 \mathrm{MH}$ & $2.20 \pm 1.40$ & $1.77 \pm 2.03$ & $2.6 \pm 1.6$ & $2.18 \pm 0.43$ \\
\hline ISO & $6.75 \pm 2.79$ & $1.58 \pm 1.21$ & $9.4 \pm 0.8$ & $1.09 \pm 0.41$ \\
\hline VAL & $6.78 \pm 1.25$ & $1.26 \pm 0.91$ & $11.3 \pm 1.4$ & $1.16 \pm 0.42$ \\
\hline 3BrTyr & $1.49 \pm 0.75$ & $1.55 \pm 0.99$ & $7.0 \pm 9.2$ & $2.43 \pm 0.57$ \\
\hline 3ClTyr & $1.02 \pm 0.82$ & $0.75 \pm 0.85$ & $2.3 \pm 2.1$ & $0.92 \pm 0.26$ \\
\hline HIS & $8.38 \pm 5.27$ & $0.82 \pm 0.57$ & $8.8 \pm 9.9$ & $0.15 \pm 0.04$ \\
\hline THRE & $7.39 \pm 0.84$ & $1.17 \pm 0.64$ & $7.1 \pm 2.4$ & $1.42 \pm 0.26$ \\
\hline TRP & $4.69 \pm 1.89$ & $0.69 \pm 0.51$ & $6.8 \pm 1.8$ & $0.99 \pm 0.27$ \\
\hline ALA & $8.42 \pm 1.39$ & $1.61 \pm 0.49$ & $9.5 \pm 1.7$ & $1.53 \pm 0.30$ \\
\hline GLU & $4.52 \pm 3.64$ & $0.97 \pm 0.95$ & $5.3 \pm 1.7$ & $1.15 \pm 0.21$ \\
\hline TYR & $2.91 \pm 1.97$ & $0.54 \pm 0.64$ & $4.5 \pm 4.6$ & $0.30 \pm 0.07$ \\
\hline SER & $13.40 \pm 4.38$ & $1.32 \pm 1.17$ & $8.7 \pm 4.7$ & $1.29 \pm 0.24$ \\
\hline ARG & $3.38 \pm 2.40$ & $1.09 \pm 1.56$ & $2.5 \pm 1.6$ & $1.60 \pm 0.27$ \\
\hline GLY & $9.09 \pm 2.03$ & $0.63 \pm 0.45$ & $8.3 \pm 2.7$ & $1.47 \pm 0.22$ \\
\hline EtNH2 & $5.80 \pm 4.35$ & $0.53 \pm 0.52$ & $7.0 \pm 0.6$ & $2.39 \pm 0.05$ \\
\hline TAU & $5.43 \pm 3.01$ & $0.89 \pm 0.46$ & $5.5 \pm 1.8$ & $1.42 \pm 0.08$ \\
\hline ASP & $4.08 \pm 3.11$ & $0.58 \pm 0.42$ & $5.1 \pm 3.3$ & $1.15 \pm 0.16$ \\
\hline LYS & $1.79 \pm 1.34$ & $0.52 \pm 0.47$ & $0.5 \pm 0.5$ & $0.37 \pm 0.15$ \\
\hline
\end{tabular}

${ }^{1}$ Mean $\%$ Response $=($ Area double blank/mean LLOQ Area $) \times 100, n($ double blank $)=6, n($ LLOQ $)=3$

${ }^{2}$ Mean $\%$ Response $=($ Area blank $/$ mean LLOQ Area $) \times 100, n($ blank $)=6, n($ LLOQ $)=3$

${ }^{3} n(\mathrm{ULOQ})=3$ 


\section{Carry over effects}

No specific recommendations are available in the FDA guidelines for the evaluation of carry over effects, therefore, the EMA guidelines were used $[35,36]$. Briefly, blank urine samples were analyzed following the injection of the ULOQ of the calibration curve as well as selected patient samples with high analyte concentrations. The responses obtained at the analyte channel in these blank solutions did not exceed $20 \%$ of that of the LLOQ indicating a negligible carry over (Supplemental Tables (Table 3)). However, during the processing of a preliminary batch of 12 patients' samples, $\underline{2}$ metabolites in $\underline{2}$ samples showed carry over effects in their subsequent blank injections. No relationship was identified between the observed carry over effects and the contained analyte concentration; in fact, the analytes were within their linear ranges. This observation can be probably explained by the late elution of residues that had been retained on the column from previously highly concentrated solutions injected over time [57].

To further avoid the rare possibility of carryover, we routinely injected blank samples after the ULOQ or HQC. Patient samples were also arranged ascendingly as per their creatinine values. The frequency of blank injection was increased towards the analysis of patient samples with high creatinine values. The autosampler needle was also washed using $5 \%$ isopropanol between the injected samples.

\section{Lower limit of quantification and linearity}

The LLOQ was determined based on the regulatory guidelines, in which, at the LLOQ, each analyte peak area mean $(n=6)$ is at least 5 times higher than that observed at the double blank [35, 36]. For validating method linearity, a set of 8 calibration standards was used to construct a 200 fold linear curve for each derivatized analyte over the concentration ranges compiled in Supplemental Tables (Table 4). At least $75 \%$ of the calibration standards; 6 points including the LLOQ and ULOQ, showed acceptable deviations from their nominal values $( \pm 20 \%$ for LLOQ, 
$\pm 15 \%$ for the other points). As seen in Supplemental Tables (Table 4), satisfactory linear relationships were achieved $\left(\mathrm{r}^{2} \geq 0.990\right)$.

Linearity was first constructed using the simple least square regression model. In order to assess the homoscedasticity of the regression analysis; an F-test for the variances of LLOQ and HQC

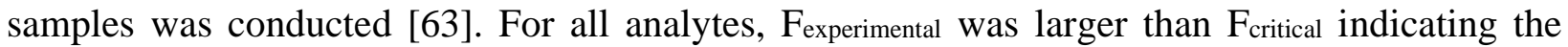
necessity of a weighing factor to correct for the influence of the standard deviation of the large concentrations on the lower ones (Supplemental Tables (Table 5)). Factors of $1 / \mathrm{x}$ and $1 / \mathrm{x}^{2}$ were tested and $\mathrm{CV} \%$ for each calculated regression concentration was computed against the nominal value. For all analytes, a factor of $1 / \mathrm{x}^{2}$ resulted in the least sum of absolute CV\% values across the whole calibration range, and consequently, $1 / \mathrm{x}^{2}$ was used for curves weighing (Supplemental Tables (Table 5)) [63].

Intra- and inter day accuracy and precision

As seen from the data gathered in Supplemental Tables (Tables 6 and 7), acceptable intra- and inter- day accuracies were demonstrated through the $\%$ recovery $(\% \mathrm{R})$ values that were between $85 \%$ and $115 \%$ at all levels, as per the FDA and EMA guidelines [35, 36]. Intra and inter-day precision was also acceptable with a CV\% less than $17 \%$ for the LLOQ and less than $15 \%$ for all other levels.

\section{$\underline{\text { Stability }}$}

Stability studies were mainly conducted to ensure that the concentrations of the derivatized metabolites are not varied under conditions that are likely to be encountered during sample processing and analysis. On a routine basis, $\underline{3}$ hours was the average sample processing time for a set of 15 patient samples along with the needed standards and QC solutions. For this reason, HQC and LQC samples (5 replicates) were processed as described and they were set aside for an 
additional $4 \mathrm{~h}$ period at room temperature. QC samples analyzed against freshly prepared calibration standards were stable for $4 \mathrm{~h}$, where their $\% \mathrm{R}$ values were between $95 \%$ and $112 \%$ and their CV\% values were less than 10\% (Supplemental Tables (Table 8)).

In addition to sample processing times, the relatively lengthy chromatographic run time necessitated the evaluation of the autosampler stability. QC samples were found stable for $48 \mathrm{~h}$ at $4{ }^{\circ} \mathrm{C}$ (Supplemental Tables (Table 8)), thus allowing for overnight collection of data from patient samples.

Freeze-thaw stability was also evaluated by exposing the prepared QC samples for 3 cycles of freezing to $-80^{\circ} \mathrm{C}$ and thawing to room temperature. Freezing time in each cycle was not less 12 h. Again, derivatized QC samples were found stable (Supplemental Tables (Table 8)). Although our standard operating procedure recommends the analysis of freshly prepared patient samples, the freeze-thaw stability of the processed samples showed that refreezing is possible during unforeseen instrument down times.

Short and long term storage stability for 2 weeks and 3 months, respectively, was also appraised. From the data gathered (Supplemental Tables (Table 8)), derivatized samples remained stable after a 2 week storage period. However, the samples were not stable when stored for a longer period of time as the accuracies ranged from $75 \%$ to $89.9 \%$. This finding was not of a concern as the derivatized samples are unlikely to be reanalyzed after a 3 month storage period. Alternatively, a freshly thawed urine sample can be analyzed if needed. Additionally, data gathered in Supplemental Tables (Table 8) for evaluating 3-month stock stability showed acceptable accuracy and precision of the analyzed QC samples. 
Finally, the stability of underivatized urine (i.e. the metabolome stability) while being stored at $80^{\circ} \mathrm{C}$, was assessed. An aliquot of a selected patient sample was thawed, processed and analyzed against a freshly prepared calibration curve once monthly over a 4 months period. The precision of the analysis for the majority of the quantified metabolites was acceptable as demonstrated by the CV\% values that were less than $15 \%$. The only metabolite outliers were arginine (15.97\%) and sarcosine (51.3\%) (Supplemental Tables (Table 8)). The unacceptable CV\% for sarcosine can be attributed to its instability on the $3^{\text {rd }}$ and $4^{\text {th }}$ months of analyses. There is a common consensus that lower temperatures of storage can preserve the metabolomic profile of biological samples. Unfortunately, a comprehensive study that compares accurate metabolomes quantities is lacking, A recent review by Khamis el al [64] provided more information on the published literature regarding urine metabolome stability under different temperatures.

\section{Dilution integrity}

Dilution integrity was tested by mixing ${ }^{12} \mathrm{C}_{2}$-derivatized analytes stock solution with blank surrogate urine matrix at ratio of 4:1. The obtained data demonstrates the acceptable accuracy and precision where the $\% \mathrm{R}$ values were within $\pm 15 \%$ and were not deviating by more than $15 \%$ (Supplemental tables (Table 9)). Dilution integrity was also evaluated at 50 and 100 fold level, however, unacceptable accuracies were usually obtained in some of the replicates leading to additional unacceptable precisions (data not shown). Nonetheless, there was not a single event during the analysis of 80 patient urine samples in which a 20 fold dilution was not sufficient to measure any of the derivatized metabolites existing outside their ULOQ.

\section{Incurred sample reanalysis}

Recent opinions suggest that despite the rigidity in validating bioanalytical methods; standards and QC samples may not entirely reflect the extent of the variation experienced in patient samples [65]. Consequently, incurred sample reanalysis has been encouraged to be included within the validation 
studies $[36,65]$. In some cases, significant differences in the concentrations of analytes were reported when study samples were reanalyzed despite the acceptance of the batch through the included QC samples [65]. Despite the absence of recommendations with the FDA, the EMA suggests that $10 \%$ of the samples should be reanalyzed if the studied samples are less than 1000 . The calculated concentrations should be within $20 \%$ of their mean in $67 \%$ of the repeats $[36,65]$.

Accordingly, for a batch of 42 patient samples, aliquots from 4 selected patient urine samples were freshly thawed on 3 different days and analyzed in duplicates (6 replicates in total). For 2 patients, the concentrations of all quantified metabolites were below $17 \%$ of their mean in at least $67 \%$ of the repeats (Table 3) meeting the specifications set by the EMA that is $20 \%$ in at least $67 \%$ [36, 65]. For 1 patient, 1 metabolite out of the 19 targets, 1-methylhistamine, showed unacceptable accuracies in $50 \%$ of the repeats specifically $(\% \mathrm{R}=71 \%, 121 \%$ and $125 \%)$. As for the last patient, percentage recoveries of alanine were possibly biased by the analyses obtained from day 3 (statistical outliers) (Table 3). Setting the distorted arithmetic mean as the reference for the calculation of $\%$ R, i.e. accuracy of alanine, resulted in values ranging from 39-135\%. In fact, these findings confirm the limitations of the available EMA guidelines where values compared to the arithmetic mean are highly likely to be influenced by outlier values. On the other hand, there have been analytical calls for using the original value as the reference value with the aim of avoiding the error induced by the skewed mean [65]. Despite the fact that the outliers represent around only $1.2 \%$ of the total measured values, the limited number of repeated patient samples remains as a limitation to that study. It should be emphasized that the remaining 18 metabolites in patients 2 and 3 were within the acceptable criteria, as set by regulatory bodies. 
Table 3: Incurred sample reanalysis for 4 patient samples using the optimized analytical method

\begin{tabular}{|c|c|c|c|c|c|c|c|c|c|c|c|c|}
\hline \multirow[t]{2}{*}{ Analyte } & \multicolumn{3}{|c|}{ Study subject 1} & \multicolumn{3}{|c|}{ Study subject 2} & \multicolumn{3}{|c|}{ Study subject 3} & \multicolumn{3}{|c|}{ Study subject 4} \\
\hline & $\begin{array}{c}\text { Found } \\
(\mathrm{ng} / \mathrm{mL}), \\
\mathrm{n}=6\end{array}$ & $\% \mathrm{R}$ & $\begin{array}{l}\mathrm{CV} \\
(\%) \\
\end{array}$ & $\begin{array}{c}\text { Found } \\
(\mathrm{ng} / \mathrm{mL}) \\
\mathrm{n}=6\end{array}$ & $\% \mathrm{R}$ & $\begin{array}{l}\mathrm{CV} \\
(\%) \\
\end{array}$ & $\begin{array}{c}\text { Found } \\
(\mathrm{ng} / \mathrm{mL}) \\
\mathrm{n}=6\end{array}$ & $\% \mathrm{R}$ & $\begin{array}{l}\mathrm{CV} \\
(\%) \\
\end{array}$ & $\begin{array}{c}\text { Found } \\
(\mathrm{ng} / \mathrm{mL}) \\
\mathrm{n}=6\end{array}$ & $\% \mathrm{R}$ & $\begin{array}{l}\mathrm{CV} \\
(\%) \\
\end{array}$ \\
\hline \multirow[t]{6}{*}{ SAR } & 265.6 & 101.53 & 4.7 & 361.6 & 101.19 & 11 & 140.8 & 106.62 & 6.11 & 104.8 & 112.54 & 12.3 \\
\hline & 256 & 97.86 & & 412.8 & 115.52 & & 137.92 & 104.44 & & 79.68 & 85.57 & \\
\hline & 241.6 & 92.35 & & 340.8 & 95.37 & & 122.4 & 92.69 & & 106.88 & 114.78 & \\
\hline & 262.4 & 100.31 & & 299.2 & 83.73 & & 123.68 & 93.66 & & 96.32 & 103.44 & \\
\hline & 278.4 & 106.42 & & 385.6 & 107.91 & & 138.56 & 104.93 & & 82.08 & 88.14 & \\
\hline & 265.6 & 101.53 & & 344 & 96.27 & & 128.96 & 97.66 & & 88.96 & 95.53 & \\
\hline \multirow{6}{*}{$1 \mathrm{MH}$} & 163.2 & 101.04 & 10.2 & 320 & 110.40 & 22.4 & 74.88 & 109.30 & 12.7 & 75.84 & 112.46 & 12.9 \\
\hline & 161.6 & 100.05 & & 363.2 & 125.30 & & 72.8 & 106.27 & & 59.68 & 88.49 & \\
\hline & 135.04 & 83.61 & & 233.6 & 80.59 & & 55.36 & 80.81 & & 79.68 & 118.15 & \\
\hline & 154.08 & 95.39 & & 204.8 & 70.65 & & 59.84 & 87.35 & & 60.32 & 89.44 & \\
\hline & 184 & 113.92 & & 352 & 121.44 & & 76.32 & 111.41 & & 60.8 & 90.15 & \\
\hline & 171.2 & 105.99 & & 265.6 & 91.63 & & 71.84 & 104.87 & & 68.32 & 101.30 & \\
\hline \multirow{6}{*}{ ISO } & 796.8 & 106.37 & 11.7 & 4592 & 108.44 & 12.8 & 486.4 & 108.12 & 10 & 323.2 & 111.91 & 9.6 \\
\hline & 769.6 & 102.74 & & 5072 & 119.77 & & 480 & 106.70 & & 267.2 & 92.52 & \\
\hline & 588.8 & 78.60 & & 3952 & 93.32 & & 406.4 & 90.34 & & 318.4 & 110.25 & \\
\hline & 710.4 & 94.84 & & 3504 & 82.75 & & 382.4 & 85.00 & & 289.6 & 100.28 & \\
\hline & 817.6 & 109.15 & & 4208 & 99.37 & & 457.6 & 101.72 & & 252.8 & 87.53 & \\
\hline & 811.2 & 108.29 & & 4080 & 96.35 & & 486.4 & 108.12 & & 281.6 & 97.51 & \\
\hline \multirow{6}{*}{ VAL } & 3264 & 105.15 & 9.6 & 12656 & 105.35 & 10.3 & 2448 & 104.79 & 6.3 & 1452.8 & 110.26 & 11.2 \\
\hline & 2896 & 93.30 & & 13824 & 115.07 & & 2448 & 104.79 & & 1228.8 & 93.26 & \\
\hline & 2640 & 85.05 & & 11520 & 95.89 & & 2240 & 95.89 & & 1550.4 & 117.67 & \\
\hline & 3056 & 98.45 & & 10544 & 87.77 & & 2112 & 90.41 & & 1265.6 & 96.05 & \\
\hline & 3376 & 108.76 & & 12624 & 105.08 & & 2480 & 106.16 & & 1214.4 & 92.17 & \\
\hline & 3392 & 109.28 & & 10912 & 90.83 & & 2288 & 97.95 & & 1193.6 & 90.59 & \\
\hline 3BrTyr & NA & & & NA & & & NA & & & NA & & \\
\hline
\end{tabular}




\begin{tabular}{|c|c|c|c|c|c|c|c|c|c|c|c|c|}
\hline 3ClTyr & NA & & & NA & & & NA & & & $\mathrm{NA}$ & & \\
\hline \multirow{6}{*}{ HIS } & 20640 & 104.59 & \multirow[t]{6}{*}{4.5} & 53760 & 88.23 & 17.82 & 30880 & 99.66 & 4.3 & 20800 & 115.38 & \multirow[t]{6}{*}{8.03} \\
\hline & 20320 & 102.97 & & 63040 & 103.46 & & 29600 & 95.52 & & 17760 & 98.52 & \\
\hline & 18080 & 91.62 & & 73120 & 120.00 & & 32480 & 104.82 & & 18240 & 101.18 & \\
\hline & 20000 & 101.35 & & 73440 & 120.53 & & 32160 & 103.79 & & 16800 & 93.20 & \\
\hline & 19840 & 100.54 & & 55360 & 90.85 & & 31520 & 101.72 & & 17120 & 94.97 & \\
\hline & 19520 & 98.92 & & 46880 & 76.94 & & 29280 & 94.49 & & 17440 & 96.75 & \\
\hline \multirow{6}{*}{ THRE } & 8032 & 104.77 & \multirow[t]{6}{*}{9.1} & 16640 & 106.67 & 13 & 6784 & 108.21 & 9.6 & 3952 & 124.64 & \multirow[t]{6}{*}{14.3} \\
\hline & 7984 & 104.14 & & 18400 & 117.95 & & 6432 & 102.59 & & 3168 & 99.92 & \\
\hline & 6432 & 83.90 & & 14048 & 90.05 & & 5888 & 93.92 & & 2816 & 88.81 & \\
\hline & 7472 & 97.46 & & 12992 & 83.28 & & 5232 & 83.45 & & 2640 & 83.26 & \\
\hline & 8480 & 110.61 & & 16480 & 105.64 & & 6736 & 107.44 & & 3216 & 101.43 & \\
\hline & 7600 & 99.13 & & 15040 & 96.41 & & 6544 & 104.38 & & 3232 & 101.93 & \\
\hline \multirow{6}{*}{ TRP } & 7088 & 106.28 & \multirow[t]{6}{*}{12} & 31040 & 103.37 & 11 & 3520 & 106.71 & 9.1 & 4272 & 119.37 & \multirow[t]{6}{*}{12.4} \\
\hline & 7024 & 105.32 & & 35360 & 117.76 & & 3504 & 106.22 & & 3632 & 101.49 & \\
\hline & 5360 & 80.37 & & 28160 & 93.78 & & 2912 & 88.28 & & 3504 & 97.91 & \\
\hline & 6112 & 91.64 & & 26560 & 88.45 & & 2912 & 88.28 & & 2912 & 81.37 & \\
\hline & 7120 & 106.76 & & 31680 & 105.51 & & 3472 & 105.25 & & 3408 & 95.23 & \\
\hline & 7312 & 109.64 & & 27360 & 91.12 & & 3472 & 105.25 & & 3744 & 104.62 & \\
\hline \multirow{6}{*}{ ALA } & 14448 & 103.87 & \multirow[t]{6}{*}{7} & 30400 & 100.71 & 9.4 & 26880 & 135.16 & 46.8 & 2944 & 118.58 & \multirow[t]{6}{*}{10} \\
\hline & 14240 & 102.38 & & 34240 & 113.43 & & 24800 & 124.70 & & 2384 & 96.03 & \\
\hline & 12032 & 86.50 & & 28000 & 92.76 & & 7776 & 39.10 & & 2576 & 103.76 & \\
\hline & 13776 & 99.04 & & 26720 & 88.52 & & 8032 & 40.39 & & 2256 & 90.87 & \\
\hline & 14720 & 105.83 & & 32640 & 108.13 & & 26720 & 134.35 & & 2336 & 94.09 & \\
\hline & 14240 & 102.38 & & 29120 & 96.47 & & 25120 & 126.31 & & 2400 & 96.67 & \\
\hline \multirow{6}{*}{ GLU } & 31520 & 108.94 & \multirow[t]{6}{*}{8.3} & 60160 & 101.39 & 12.4 & 31360 & 111.68 & 7.6 & 20640 & 124.20 & \multirow[t]{6}{*}{14.4} \\
\hline & 29920 & 103.41 & & 69600 & 117.30 & & 28480 & 101.42 & & 17600 & 105.91 & \\
\hline & 25280 & 87.37 & & 52160 & 87.91 & & 26720 & 95.16 & & 15536 & 93.49 & \\
\hline & 27360 & 94.56 & & 50400 & 84.94 & & 24960 & 88.89 & & 13456 & 80.97 & \\
\hline & 31200 & 107.83 & & 65120 & 109.75 & & 28320 & 100.85 & & 16320 & 98.20 & \\
\hline & 28320 & 97.88 & & 58560 & 98.70 & & 28640 & 101.99 & & 16160 & 97.24 & \\
\hline
\end{tabular}




\begin{tabular}{|c|c|c|c|c|c|c|c|c|c|c|c|c|}
\hline \multirow{6}{*}{ TYR } & 8896 & 105.97 & 7.4 & 21120 & 91.24 & 7.8 & 5232 & 105.48 & 4.4 & 4752 & 117.47 & 12.1 \\
\hline & 8528 & 101.59 & & 23200 & 100.23 & & 4864 & 98.06 & & 3808 & 94.13 & \\
\hline & 7168 & 85.39 & & 24480 & 105.76 & & 5216 & 105.16 & & 4576 & 113.12 & \\
\hline & 8480 & 101.02 & & 25760 & 111.29 & & 4944 & 99.68 & & 3568 & 88.20 & \\
\hline & 8768 & 104.45 & & 23040 & 99.54 & & 4800 & 96.77 & & 3776 & 93.34 & \\
\hline & 8528 & 101.59 & & 21280 & 91.94 & & 4704 & 94.84 & & 3792 & 93.74 & \\
\hline \multirow{6}{*}{ SER } & 21280 & 105.98 & 5.6 & 42720 & 104.23 & 12.3 & 13168 & 106.26 & 5.5 & 7840 & 113.91 & 10 \\
\hline & 19840 & 98.80 & & 48960 & 119.45 & & 12320 & 99.42 & & 7296 & 106.01 & \\
\hline & 18080 & 90.04 & & 37280 & 90.96 & & 11744 & 94.77 & & 6432 & 93.45 & \\
\hline & 19840 & 98.80 & & 34720 & 84.71 & & 11584 & 93.48 & & 5872 & 85.32 & \\
\hline & 20800 & 103.59 & & 43040 & 105.01 & & 13216 & 106.65 & & 6784 & 98.57 & \\
\hline & 20640 & 102.79 & & 39200 & 95.64 & & 12320 & 99.42 & & 7072 & 102.75 & \\
\hline \multirow{6}{*}{$\mathrm{ARG}$} & 2176 & 105.02 & 7.4 & 5408 & 98.59 & 15.9 & 1904 & 105.62 & 4.5 & 1209.6 & 123.80 & 14 \\
\hline & 2288 & 110.42 & & 6720 & 122.51 & & 1824 & 101.18 & & 955.2 & 97.76 & \\
\hline & 1840 & 88.80 & & 5056 & 92.17 & & 1696 & 94.08 & & 816 & 83.52 & \\
\hline & 2048 & 98.84 & & 4560 & 83.13 & & 1728 & 95.86 & & 891.2 & 91.21 & \\
\hline & 2000 & 96.53 & & 6368 & 116.09 & & 1872 & 103.85 & & 974.4 & 99.73 & \\
\hline & 2080 & 100.39 & & 4800 & 87.51 & & 1792 & 99.41 & & 1016 & 103.98 & \\
\hline \multirow{6}{*}{ GLY } & 42560 & 102.64 & 5.1 & 71200 & 96.81 & 7.2 & 39040 & 107.65 & 5.9 & 32480 & 109.63 & 6.7 \\
\hline & 41120 & 99.16 & & 81600 & 110.95 & & 36640 & 101.03 & & 28000 & 94.51 & \\
\hline & 38080 & 91.83 & & 71680 & 97.46 & & 34720 & 95.74 & & 31680 & 106.93 & \\
\hline & 41120 & 99.16 & & 68320 & 92.89 & & 32960 & 90.88 & & 29280 & 98.83 & \\
\hline & 44480 & 107.27 & & 78560 & 106.82 & & 37280 & 102.79 & & 27680 & 93.43 & \\
\hline & 41440 & 99.94 & & 69920 & 95.07 & & 36960 & 101.91 & & 28640 & 96.67 & \\
\hline \multirow{6}{*}{ EtNH2 } & 32320 & 102.62 & 4.5 & 68480 & 103.30 & 9.5 & 19360 & 108.68 & 5.3 & 8560 & 115.26 & 11.3 \\
\hline & 31200 & 99.07 & & 75040 & 113.19 & & 18240 & 102.40 & & 6864 & 92.42 & \\
\hline & 28960 & 91.96 & & 65280 & 98.47 & & 16960 & 95.21 & & 8304 & 111.81 & \\
\hline & 31200 & 99.07 & & 57280 & 86.40 & & 16960 & 95.21 & & 7472 & 100.61 & \\
\hline & 32800 & 104.15 & & 69920 & 105.47 & & 18080 & 101.50 & & 6560 & 88.33 & \\
\hline & 32480 & 103.13 & & 61760 & 93.16 & & 17280 & 97.01 & & 6800 & 91.56 & \\
\hline TAU & 5024 & 98.69 & 6 & 116800 & 101.91 & 8.2 & 37440 & 107.75 & 5.5 & 26560 & 117.45 & 11 \\
\hline
\end{tabular}




\begin{tabular}{|c|c|c|c|c|c|c|c|c|c|c|c|c|}
\hline & 5264 & 103.40 & & 127840 & 111.54 & & 35680 & 102.69 & & 20640 & 91.27 & \\
\hline & 4672 & 91.78 & & 111360 & 97.16 & & 33120 & 95.32 & & 24800 & 109.67 & \\
\hline & 4944 & 97.12 & & 100800 & 87.95 & & 32480 & 93.48 & & 21920 & 96.93 & \\
\hline & 5568 & 109.38 & & 120960 & 105.54 & & 36000 & 103.61 & & 20960 & 92.69 & \\
\hline & 5072 & 99.63 & & 109920 & 95.91 & & 33760 & 97.16 & & 20800 & 91.98 & \\
\hline \multirow{6}{*}{ ASP } & 7408 & 105.63 & 6.6 & 15584 & 103.34 & 16.1 & 7152 & 106.73 & 7.8 & 4592 & 124.69 & 15.4 \\
\hline & 7280 & 103.80 & & 17920 & 118.83 & & 6864 & 102.43 & & 3648 & 99.06 & \\
\hline & 6256 & 89.20 & & 13088 & 86.79 & & 5936 & 88.58 & & 3216 & 87.33 & \\
\hline & 6656 & 94.90 & & 11552 & 76.60 & & 6192 & 92.40 & & 2960 & 80.38 & \\
\hline & 7360 & 104.94 & & 17280 & 114.59 & & 7200 & 107.44 & & 3856 & 104.71 & \\
\hline & 7120 & 101.52 & & 15056 & 99.84 & & 6864 & 102.43 & & 3824 & 103.84 & \\
\hline \multirow{6}{*}{ LYS } & 2032 & 109.04 & 12.5 & 7408 & 102.43 & 13.5 & 8816 & 111.16 & 10.7 & 6144 & 108.32 & 14.2 \\
\hline & 1952 & 104.75 & & 8560 & 118.36 & & 8368 & 105.51 & & 4960 & 87.45 & \\
\hline & 1421 & 76.25 & & 6928 & 95.80 & & 7152 & 90.18 & & 6928 & 122.14 & \\
\hline & 1792 & 96.16 & & 5840 & 80.75 & & 6768 & 85.34 & & 5888 & 103.81 & \\
\hline & 2016 & 108.18 & & 8016 & 110.84 & & 8736 & 110.15 & & 4784 & 84.34 & \\
\hline & 1968 & 105.61 & & 6640 & 91.81 & & 7744 & 97.65 & & 5328 & 93.94 & \\
\hline
\end{tabular}




\subsection{Analysis of patient samples}

Figures $3 \mathrm{~A}$ and $3 \mathrm{~B}$ are representative extracted ion chromatograms obtained from urine sample analyzed from asthmatic and COPD patients, respectively. Histidine and glutamine, in most of the samples, were present at concentrations above their ULOQ and sample dilution was required for their quantification. Occasionally (less than $30 \%$ of the studied samples) alanine and taurine quantification needed sample dilution as well, and rare cases of out-of-the range high concentrations (less than 5\% of the samples) were observed for tryptophan, valine, ethanolamine and threonine. One interesting finding is that creatinine concentration was not always indicative of other metabolite concentration despite having a constant output/day/subject [66]. For bromo and chloro-tyrosine, both of them were not detected within all patient samples. This agrees well with the literature in which specific sample pre-concentration and extraction steps were needed for their quantification $[67,68]$.

Patient urine samples were analyzed by the developed methodology and data was analyzed using partial least square discriminant analysis (PLS-DA). Blinded asthma and COPD samples analyzed using SIMCA ${ }^{\circledR}$ software (Umetrics, Sweden) were adequately assigned with their correct diagnosis based on the concentration of metabolites included in the training set (data not shown). The altered metabolites identified as the final biomarkers for differential diagnosis of asthma and COPD along with the involved biochemical pathways is outside the scope of this work, however, data generated from all metabolites previously identified from the ${ }^{1} \mathrm{H}-\mathrm{NMR}$ will be compiled in a separate research article focusing on the clinical relevance. 


\section{Conclusion}

An accurate, sensitive and validated method for the quantification of 19 urinary metabolites bearing amino and/or phenolic groups is successfully developed. The DIL approach is used so that conventional $\mathrm{C}_{18}$ column is applied with significant enhancement in ionization. Dansyl chloride was an excellent choice for derivatization as it allowed for the generation of an internal standard for each metabolite addressing matrix effects and any variations resulting from sample preparations. During method validation, key challenges were observed, particularly in the optimization the linear ranges for each metabolite in patient urine samples. Validation of selectivity was also halted by the presence of isotopic contribution from the light derivatized metabolites.

The statistical analysis using partial least square discriminant analysis (PLS-DA) from the dansylated metabolites will be combined with the results obtained from the remaining 50 target metabolites. The results from patient samples and the identified biomarkers for the differential diagnosis of asthma and COPD will be published in a separate research article where more emphasis on clinical aspect of the findings is discussed.

In summary, we have demonstrated the combination of DIL with LC-MS/MS analysis for targeted biomarker discovery. This technique can allow the development and validation of robust analytical methods needed for biomarkers quantification prior to FDA submission. By adopting this methodology, different submetabolome of carboxylic acids [58], alcohols [69] and thiols [70] can be targeted and quantified. 


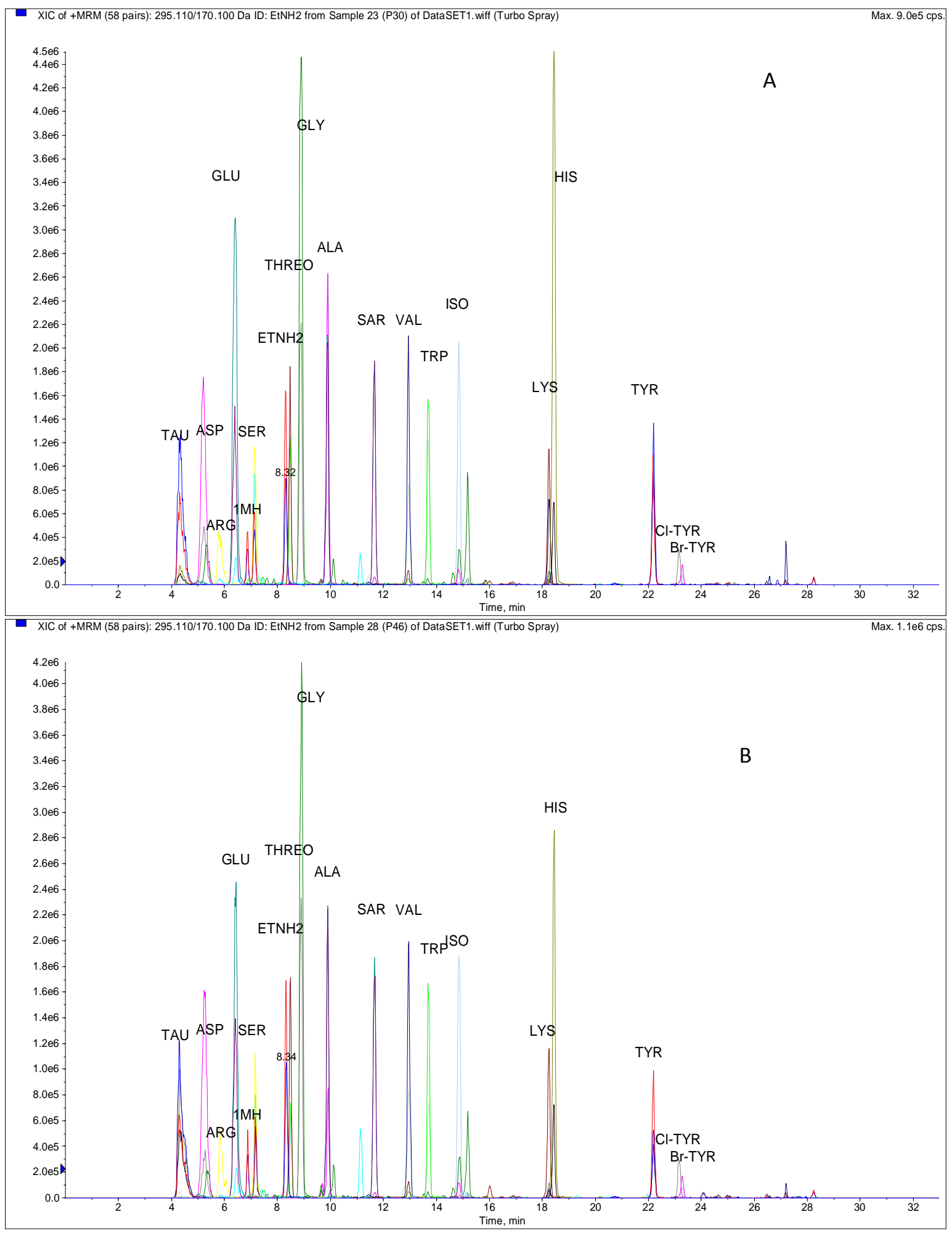

Figure 3: Extracted ion chromatogram of derivatized metabolites quantified in A) asthma and B) COPD patient urine sample. 


\section{Acknowledgment}

The authors acknowledge funding form Saskatchewan Health Research Foundation and AllerGen NCE Inc. (AllerGen). Funding for the purchase of the ABSciex QTRAP 4000 instrument was obtained through a Canada Foundation for Innovation grant- Leaders Opportunity Fund. We also acknowledge Dr. Liang Li, University of Alberta, for valuable discussions and for providing labeled DNS-Cl for initial experiments. Ms. Hanan Awad, Dr.

Randy Purves and Ms. Deborah Michel are acknowledged for their technical help and valuable discussions. Dr. Kenneth Nelson is acknowledged for his assistance on the synthesis of DNS-Cl reagents used in this study. Dr. George Katselis is acknowledged for his feedback to the manuscript contents. Ms. Khamis thanks the College of Pharmacy and Nutrition for scholarship funding. 


\section{References}

[1] A.A. Cruz, J. Bousquet, N. Khaltaev, Global surveillance, prevention and control of chronic respiratory diseases: a comprehensive approach, World Health Organization2007.

[2] P. Gibson, J. Simpson, The overlap syndrome of asthma and COPD: what are its features and how important is it?, Thorax, 64 (2009) 728-735.

[3] E.G. Tzortzaki, A. Proklou, N.M. Siafakas, Asthma in the elderly: can we distinguish it from COPD?, Journal of allergy, 2011 (2011).

[4] J. Chang, Z. Mosenifar, Differentiating COPD from asthma in clinical practice, Journal of intensive care medicine, 22 (2007) 300-309.

[5] R.H. Green, C.E. Brightling, S. McKenna, B. Hargadon, D. Parker, P. Bradding, A.J. Wardlaw, I.D.

Pavord, Asthma exacerbations and sputum eosinophil counts: a randomised controlled trial, The Lancet, 360 (2002) 1715-1721.

[6] H.L. Petsky, C.J. Cates, A. Li, J.A. Kynaston, C. Turner, A.B. Chang, Tailored interventions based on exhaled nitric oxide versus clinical symptoms for asthma in children and adults, Cochrane Database Syst Rev, 4 (2009).

[7] L. Fromer, C.B. Cooper, A review of the GOLD guidelines for the diagnosis and treatment of patients with COPD, International journal of clinical practice, 62 (2008) 1219-1236.

[8] D.G. Tinkelman, D.B. Price, R.J. Nordyke, R. Halbert, Misdiagnosis of COPD and asthma in primary care patients 40 years of age and over, Journal of Asthma, 43 (2006) 75-80.

[9] S. Snowden, S.-E. Dahlén, C.E. Wheelock, Application of metabolomics approaches to the study of respiratory diseases, Bioanalysis, 4 (2012) 2265-2290.

[10] B.F. Nobakht M. Gh, R. Aliannejad, M. Rezaei-Tavirani, S. Taheri, A.A. Oskouie, The metabolomics of airway diseases, including COPD, asthma and cystic fibrosis, Biomarkers, 20 (2015) 5-16.

[11] A. Zhang, H. Sun, X. Wu, X. Wang, Urine metabolomics, Clinica Chimica Acta, 414 (2012) 65-69.

[12] W.B. Dunn, D.I. Broadhurst, H.J. Atherton, R. Goodacre, J.L. Griffin, Systems level studies of mammalian metabolomes: the roles of mass spectrometry and nuclear magnetic resonance spectroscopy, Chemical Society Reviews, 40 (2011) 387-426.

[13] R.D. Beger, T. Colatsky, Metabolomics data and the biomarker qualification process, Metabolomics, 8 (2012) 2-7.

[14] M. Mamas, W.B. Dunn, L. Neyses, R. Goodacre, The role of metabolites and metabolomics in clinically applicable biomarkers of disease, Archives of toxicology, 85 (2011) 5-17.

[15] H. Wu, R. Xue, L. Dong, T. Liu, C. Deng, H. Zeng, X. Shen, Metabolomic profiling of human urine in hepatocellular carcinoma patients using gas chromatography/mass spectrometry, Analytica chimica acta, 648 (2009) 98-104.

[16] H.M. Woo, K.M. Kim, M.H. Choi, B.H. Jung, J. Lee, G. Kong, S.J. Nam, S. Kim, S.W. Bai, B.C. Chung, Mass spectrometry based metabolomic approaches in urinary biomarker study of women's cancers, Clinica Chimica Acta, 400 (2009) 63-69.

[17] H.J. Issaq, O. Nativ, T. Waybright, B. Luke, T.D. Veenstra, E.J. Issaq, A. Kravstov, M. Mullerad, Detection of bladder cancer in human urine by metabolomic profiling using high performance liquid chromatography/mass spectrometry, The Journal of urology, 179 (2008) 2422-2426.

[18] K. Kim, P. Aronov, S.O. Zakharkin, D. Anderson, B. Perroud, I.M. Thompson, R.H. Weiss, Urine metabolomics analysis for kidney cancer detection and biomarker discovery, Molecular \& cellular proteomics, 8 (2009) 558-570.

[19] X. Wang, A. Zhang, Y. Han, P. Wang, H. Sun, G. Song, T. Dong, Y. Yuan, X. Yuan, M. Zhang, Urine metabolomics analysis for biomarker discovery and detection of jaundice syndrome in patients with liver disease, Molecular \& Cellular Proteomics, 11 (2012) 370-380. 
[20] A. Lafaye, C. Junot, B.R.L. Gall, P. Fritsch, J.C. Tabet, E. Ezan, Metabolite profiling in rat urine by liquid chromatography/electrospray ion trap mass spectrometry. Application to the study of heavy metal toxicity, Rapid Communications in Mass Spectrometry, 17 (2003) 2541-2549.

[21] S. Zheng, M. Yu, X. Lu, T. Huo, L. Ge, J. Yang, C. Wu, F. Li, Urinary metabonomic study on biochemical changes in chronic unpredictable mild stress model of depression, Clinica Chimica Acta, 411 (2010) 204-209.

[22] R.D. Beger, R.D. Holland, J. Sun, L.K. Schnackenberg, P.C. Moore, C.L. Dent, P. Devarajan, D. Portilla, Metabonomics of acute kidney injury in children after cardiac surgery, Pediatric Nephrology, 23 (2008) 977-984.

[23] D.A. Rodríguez, G. Alcarraz-Vizán, S. Díaz-Moralli, M. Reed, F.P. Gómez, F. Falciani, U. Günther, J. Roca, M. Cascante, Plasma metabolic profile in COPD patients: effects of exercise and endurance training, Metabolomics, 8 (2012) 508-516.

[24] I. Bertini, C. Luchinat, M. Miniati, S. Monti, L. Tenori, Phenotyping COPD by 1 H NMR metabolomics of exhaled breath condensate, Metabolomics, 10 (2014) 302-311.

[25] B.K. Ubhi, J.H. Riley, P.A. Shaw, D.A. Lomas, R. Tal-Singer, W. MacNee, J.L. Griffin, S.C. Connor, Metabolic profiling detects biomarkers of protein degradation in COPD patients, European Respiratory Journal, 40 (2012) 345-355.

[26] B.K. Ubhi, K.K. Cheng, J. Dong, T. Janowitz, D. Jodrell, R. Tal-Singer, W. MacNee, D.A. Lomas, J.H. Riley, J.L. Griffin, Targeted metabolomics identifies perturbations in amino acid metabolism that subclassify patients with COPD, Molecular BioSystems, 8 (2012) 3125-3133.

[27] J. Jung, S.H. Kim, H.S. Lee, G. Choi, Y.S. Jung, D. Ryu, H.S. Park, G.S. Hwang, Serum metabolomics reveals pathways and biomarkers associated with asthma pathogenesis, Clinical \& Experimental Allergy, 43 (2013) 425-433.

[28] E.J. Saude, C.D. Skappak, S. Regush, K. Cook, A. Ben-Zvi, A. Becker, R. Moqbel, B.D. Sykes, B.H. Rowe, D.J. Adamko, Metabolomic profiling of asthma: diagnostic utility of urine nuclear magnetic resonance spectroscopy, Journal of Allergy and Clinical Immunology, 127 (2011) 757-764. e756.

[29] E.J. Saude, I.P. Obiefuna, R.L. Somorjai, F. Ajamian, C. Skappak, T. Ahmad, B.K. Dolenko, B.D. Sykes, R. Moqbel, D.J. Adamko, Metabolomic biomarkers in a model of asthma exacerbation: urine nuclear magnetic resonance, American journal of respiratory and critical care medicine, 179 (2009) 25-34. [30] J.L. McClay, D.E. Adkins, N.G. Isern, T.M. O'Connell, J.B. Wooten, B.K. Zedler, M.S. Dasika, B.T. Webb, B.-J. Webb-Robertson, J.G. Pounds, $1 \mathrm{H}$ nuclear magnetic resonance metabolomics analysis identifies novel urinary biomarkers for lung function, Journal of proteome research, 9 (2010) 3083-3090. [31] L. Wang, Y. Tang, S. Liu, S. Mao, Y. Ling, D. Liu, X. He, X. Wang, Metabonomic profiling of serum and urine by $1 \mathrm{H}$ NMR-based spectroscopy discriminates patients with chronic obstructive pulmonary disease and healthy individuals, PloS one, 8 (2013) e65675.

[32] C.C. Loureiro, I.F. Duarte, J. Gomes, J. Carrola, A.S. Barros, A.M. Gil, J. Bousquet, A.T. Bom, S.M. Rocha, Urinary metabolomic changes as a predictive biomarker of asthma exacerbation, Journal of Allergy and Clinical Immunology, 133 (2014) 261.

[33] C.C. Loureiro, A.S. Oliveira, M. Santos, A. Rudnitskaya, A. Todo-Bom, J. Bousquet, S.M. Rocha, Urinary metabolomic profiling of asthmatics can be related to clinical characteristics, Allergy, 71 (2016) 1362-1365.

[34] E. Mattarucchi, E. Baraldi, C. Guillou, Metabolomics applied to urine samples in childhood asthma; differentiation between asthma phenotypes and identification of relevant metabolites, Biomedical Chromatography, 26 (2012) 89-94.

[35] Food and Drug Administration, FDA guidance for industry: bioanalytical method validation, US Department of Health and Human Services, Food and Drug Administration, Center for Drug Evaluation and Research: Rockville, MD, (2001). 
[36] Eurapean Meciecnes Agency (EMA), Committee for Medicinal Products for Human Use (CHMP), Guideline on Bioanalytical Method Validation, (2011).

[37] J.W. Lee, R.S. Weiner, J.M. Sailstad, R.R. Bowsher, D.W. Knuth, P.J. O’Brien, J.L. Fourcroy, R. Dixit, L. Pandite, R.G. Pietrusko, Method validation and measurement of biomarkers in nonclinical and clinical samples in drug development: a conference report, Pharmaceutical research, 22 (2005) 499-511.

[38] D.J. Adamko, P. Nair, I. Mayers, R.T. Tsuyuki, S. Regush, B.H. Rowe, Metabolomic profiling of asthma and chronic obstructive pulmonary disease: A pilot study differentiating diseases, Journal of Allergy and Clinical Immunology, 136 (2015) 571-580. e573.

[39] K. Guo, L. Li, Differential 12C-/13C-isotope dansylation labeling and fast liquid chromatography/mass spectrometry for absolute and relative quantification of the metabolome, Analytical chemistry, 81 (2009) 3919-3932.

[40] F. Xu, L. Zou, Y. Liu, Z. Zhang, C.N. Ong, Enhancement of the capabilities of liquid chromatographymass spectrometry with derivatization: general principles and applications, Mass spectrometry reviews, 30 (2011) 1143-1172.

[41] T. Toyo'oka, LC-MS determination of bioactive molecules based upon stable isotope-coded derivatization method, Journal of pharmaceutical and biomedical analysis, 69 (2012) 174-184.

[42] R. Zhou, K. Guo, L. Li, 5-Diethylamino-naphthalene-1-sulfonyl Chloride (DensCl): A novel triplex isotope labeling reagent for quantitative metabolome analysis by liquid chromatography mass spectrometry, Analytical chemistry, 85 (2013) 11532-11539.

[43] J.R. Delanghe, M.M. Speeckaert, Creatinine determination according to Jaffe-what does it stand for?, NDT plus, (2011) sfq211.

[44] Q.C.A. Kit, https://www.bioassaysys.com/Creatinine-Assay-Kit.html.

[45] F. Bergmann, W. Pfleiderer, Nucleotides. Part XLI. The 2-dansylethoxycarbonyl (?] 2-\{[5-

(dimethylamino) naphthalen-1-yl] sulfonyl\} ethoxycarbonyl; dnseoc) group for protection of the 5'hydroxy function in oligodeoxyribonucleotide synthesis, Helvetica chimica acta, 77 (1994) 203-215.

[46] A. Mendel, Improved preparation of 5-dimethylamino-1-naphthalenesulfonyl chloride, Journal of Chemical and Engineering Data, 15 (1970) 340-341.

[47] J. Zheng, L. Li, Fragmentation of protonated dansyl-labeled amines for structural analysis of aminecontaining metabolites, International Journal of Mass Spectrometry, 316 (2012) 292-299.

[48] B. Matuszewski, M. Constanzer, C. Chavez-Eng, Strategies for the assessment of matrix effect in quantitative bioanalytical methods based on HPLC-MS/MS, Analytical chemistry, 75 (2003) 3019-3030.

[49] V.P. Shah, K.K. Midha, J.W. Findlay, H.M. Hill, J.D. Hulse, I.J. McGilveray, G. McKay, K.J. Miller, R.N.

Patnaik, M.L. Powell, Bioanalytical method validation-a revisit with a decade of progress,

Pharmaceutical research, 17 (2000) 1551-1557.

[50] C.J. Briscoe, M.R. Stiles, D.S. Hage, System suitability in bioanalytical LC/MS/MS, Journal of pharmaceutical and biomedical analysis, 44 (2007) 484-491.

[51] N. Seiler, Use of the dansyl reaction in biochemical analysis, Methods of Biochemical Analysis, Volume 18, (1970) 259-337.

[52] E. Stokvis, H. Rosing, L. López-Lázaro, J. Schellens, J. Beijnen, Switching from an analogous to a stable isotopically labeled internal standard for the LC-MS/MS quantitation of the novel anticancer drug Kahalalide F significantly improves assay performance, Biomedical Chromatography, 18 (2004) 400-402.

[53] Z. Lei, D.V. Huhman, L.W. Sumner, Mass spectrometry strategies in metabolomics, Journal of Biological Chemistry, 286 (2011) 25435-25442.

[54] F. Mazzotti, H. Benabdelkamel, L.D. Donna, C.M. Athanassopoulos, A. Napoli, G. Sindona, Light and heavy dansyl reporter groups in food chemistry: amino acid assay in beverages, Journal of Mass Spectrometry, 47 (2012) 932-939. 
[55] S. Ito, K. Tsukada, Matrix effect and correction by standard addition in quantitative liquid chromatographic-mass spectrometric analysis of diarrhetic shellfish poisoning toxins, Journal of Chromatography A, 943 (2002) 39-46.

[56] M. Jemal, A. Schuster, D.B. Whigan, Liquid chromatography/tandem mass spectrometry methods for quantitation of mevalonic acid in human plasma and urine: method validation, demonstration of using a surrogate analyte, and demonstration of unacceptable matrix effect in spite of use of a stable isotope analog internal standard, Rapid Communications in Mass Spectrometry, 17 (2003) 1723-1734. [57] C. Viswanathan, S. Bansal, B. Booth, A.J. DeStefano, M.J. Rose, J. Sailstad, V.P. Shah, J.P. Skelly, P.G. Swann, R. Weiner, Quantitative bioanalytical methods validation and implementation: best practices for chromatographic and ligand binding assays, Pharmaceutical research, 24 (2007) 1962-1973.

[58] A. Stanislaus, K. Guo, L. Li, Development of an isotope labeling ultra-high performance liquid chromatography mass spectrometric method for quantification of acylglycines in human urine, Analytica chimica acta, 750 (2012) 161-172.

[59] J.H. Zar, Biostatistical Analysis, Prentice Hall1999.

[60] Q.A. Xu, T.L. Madden, LC-MS in drug bioanalysis, Springer Science \& Business Media2012.

[61] D.J. Borts, L.D. Bowers, Direct measurement of urinary testosterone and epitestosterone conjugates using high-performance liquid chromatography/tandem mass spectrometry, Journal of mass spectrometry, 35 (2000) 50-61.

[62] L.B. Fay, S. Métairon, M. Baumgartner, Linearization of second-order calibration curves in stable isotope dilution-mass spectrometry, Flavour and fragrance journal, 16 (2001) 164-168.

[63] A.M.d. Almeida, M.M. Castel-Branco, A. Falcao, Linear regression for calibration lines revisited: weighting schemes for bioanalytical methods, Journal of Chromatography B, 774 (2002) 215-222.

[64] M.M. Khamis, D.J. Adamko, A. El-Aneed, Mass spectrometric based approaches in urine metabolomics and biomarker discovery, Mass spectrometry reviews, (2015).

[65] P. Timmerman, S. Luedtke, P. van Amsterdam, M. Brudny-Kloeppel, B. Lausecker, S. Fischmann, S. Globig, C.-J. Sennbro, J.M. Jansat, H. Mulder, Incurred sample reproducibility: views and recommendations by the European Bioanalysis Forum, Bioanalysis, 1 (2009) 1049-1056.

[66] B.M. Warrack, S. Hnatyshyn, K.-H. Ott, M.D. Reily, M. Sanders, H. Zhang, D.M. Drexler, Normalization strategies for metabonomic analysis of urine samples, Journal of Chromatography B, 877 (2009) 547-552.

[67] H.-J.C. Chen, W.-L. Chiu, Simultaneous detection and quantification of 3-nitrotyrosine and 3bromotyrosine in human urine by stable isotope dilution liquid chromatography tandem mass spectrometry, Toxicology letters, 181 (2008) 31-39.

[68] H. Mita, N. Higashi, M. Taniguchi, A. Higashi, Y. Kawagishi, K. Akiyama, Urinary 3-bromotyrosine and 3-chlorotyrosine concentrations in asthmatic patients: lack of increase in 3-bromotyrosine concentration in urine and plasma proteins in aspirin-induced asthma after intravenous aspirin challenge, Clinical \& Experimental Allergy, 34 (2004) 931-938.

[69] S. Zhao, X. Luo, L. Li, Chemical Isotope Labeling LC-MS for High Coverage and Quantitative Profiling of the Hydroxyl Submetabolome in Metabolomics, Analytical Chemistry, (2016).

[70] P. Liu, Y.-Q. Huang, W.-J. Cai, B.-F. Yuan, Y.-Q. Feng, Profiling of thiol-containing compounds by stable isotope labeling double precursor ion scan mass spectrometry, Analytical chemistry, 86 (2014) 9765-9773. 